\title{
Modality-Specific Impairment of Hippocampal CA1 Neurons of Alzheimer's Disease Model Mice
}

\author{
${ }^{(1)}$ Risa Takamura, ${ }^{1,2,3,4}$ Kotaro Mizuta, ${ }^{1,4 *}$ Yukiko Sekine, ${ }^{3,4}$ Tanvir Islam, ${ }^{3,4}$ Takashi Saito, ${ }^{3,4}$ Masaaki Sato, ${ }^{3,4,5}$ \\ Masamichi Ohkura, ${ }^{5}$ Junichi Nakai, ${ }^{5}{ }^{(1}$ Toshio Ohshima, ${ }^{2}$ Takaomi C. Saido, ${ }^{3,4}$ and ${ }^{\circledR}$ Yasunori Hayashi ${ }^{1,4,5}$ \\ ${ }^{1}$ Department of Pharmacology, Kyoto University Graduate School of Medicine, Kyoto 606-8501, Japan, ${ }^{2}$ Department of Life Science and Medical \\ Bioscience, Graduate School of Advanced Science and Engineering, Waseda University, Tokyo 162-8480, Japan, ${ }^{3}$ Center for Brain Science, RIKEN, \\ Saitama 351-0198, Japan, ${ }^{4}$ Brain Science Institute, RIKEN, Saitama 351-0198, Japan, and ${ }^{5}$ Brain and Body System Science Institute, Saitama \\ University, Saitama 338-8570, Japan
}

Impairment of episodic memory, a class of memory for spatiotemporal context of an event, is an early symptom of Alzheimer's disease. Both spatial and temporal information are encoded and represented in the hippocampal neurons, but how these representations are impaired under amyloid $\beta(\mathrm{A} \beta)$ pathology remains elusive. We performed chronic imaging of the hippocampus in awake male amyloid precursor protein $(A p p)$ knock-in mice behaving in a virtual reality environment to simultaneously monitor spatiotemporal representations and the progression of $\mathrm{A} \beta$ depositions. We found that temporal representation is preserved, whereas spatial representation is significantly impaired in the App knock-in mice. This is because of the overall reduction of active place cells, but not time cells, and compensatory hyperactivation of remaining place cells near $\mathrm{A} \boldsymbol{\beta}$ aggregates. These results indicate the differential impact of $\mathrm{A} \boldsymbol{\beta}$ aggregates on two major modalities of episodic memory, suggesting different mechanisms for forming and maintaining these two representations in the hippocampus.

Key words: Alzheimer's disease; calcium imaging; place cell; time cell; virtual reality

Significance Statement

Spatiotemporal memory impairments are common at the early stage of Alzheimer's disease patients. We demonstrate the different impairment patterns of place and time cells in the dorsal hippocampus of head-fixed App knock-in mouse by in vivo two-photon calcium imaging over months under the virtual reality spatiotemporal tasks. These results highlight that place cells were preferentially and gradually damaged by nearby $\mathrm{A} \beta$ aggregates, whereas time cells were less vulnerable. We further show these impairments were because of neuronal hyperactivity that occurs near the $\mathrm{A} \beta$ deposition. We suggest the differential and gradual impairment in two major modalities of episodic memory under $\mathrm{A} \beta$ pathology.

\section{Introduction}

Alzheimer's disease (AD) is the most common type of dementia characterized by gradual memory dysfunctions. One early clinical symptom of $\mathrm{AD}$ is the impairment of episodic memory, a memory of the place and time of an event (Morris et al., 1982; Ergorul and Eichenbaum, 2004; Carrasco et al., 2000; Kraus et

\section{Received Jan. 28, 2021; revised Apr. 10, 2021; accepted Apr. 23, 2021}

Author contributions: K.M. and Y.H. designed research; R.T., K.M., and Y.S. performed research; T.S., M.O., J.N., and T.C.S. contributed unpublished reagents/analytic tools; R.T., K.M., T.I., and M.S. analyzed data; and R.T., K.M., T.O., and Y.H. wrote the paper.

This work was supported by RIKEN; National Insitutes of Health Grant R01DA17310; KAKENHI Grants JP22110006, JP90466037, and JP18H05434; a Human Frontier Science Program grant to Y.H.; KAKENHI Grant JP20J13700 to R.T.; Inamori Foundation and KAKENHI grants JP25830023, JP15H01571, JP17H05695 and JP19K16293 to K.M.; KAKENHI Grants JP26115504, 25111703, and JP21115504 to J.N.; Regional Innovation Cluster Program Grant (City Area Type, Central Saitama Area) from the Ministry of Education, Culture, Sports, Science and Technology (MEXT) to J.N. and M.0. We thank Dr. Adam Z. Weitemier, Dr. Steven Middleton, and Dr. Kei Igarashi for comments on the manuscript, and Dr. Shigeyoshi Fujisawa, Dr. Thomas J. McHugh, and Dr. Alexander Schmidt for discussions. al., 2013; Moodley et al., 2015). The hippocampus is a crucial brain region for the formation of episodic memory. In the hippocampus, place cells encode an animal's position by firing at a specific location and provide the basic elements for forming a cognitive map of a context (O'Keefe and Dostrovsky, 1971; O’Keefe and Nadel, 1978; Cacucci et al., 2008; Zhao et al., 2014;

J. Nakai's present address: Department of Oral Physiology, Tohoku University Graduate School of Dentistry, Miyagi 980-8575, Japan.

M. Ohkura's present address: Graduate School of Pharmacy, Kyushu University of Health and Welfare, Miyazaki 882-9508, Japan.

T. Saito's present address: Department of Neurocognitive Science, Institute of Brain Science, Nagoya City University, Nagoya, Aichi 467-8601, Japan.

M. Sato's present address: Department of Neuropharmacology, Hokkaido University Graduate School of Medicine, Sapporo, Hokkaido 060-8638, Japan.

${ }^{*}$ R.T. and K.M. are co-first authors.

Y.H. was supported in part by Takeda Pharmaceutical Company, Fujitsu Laboratories, and DWANGO Correspondence should be addressed to Yasunori Hayashi at yhayashi-tky@umin.ac.jp.

https://doi.org/10.1523/JNEUROSCI.0208-21.2021

Copyright $\odot 2021$ the authors 
Cayzac et al., 2015; Mably et al., 2017). The hippocampus also bears time cells that fire sequentially in a temporally defined task in context-specific manner (Pastalkova et al., 2008) and are implicated in the perception of seconds-order time intervals (MacDonald et al., 2011, 2013). At this point, it is not clear whether time cells are involved in memory of episodes that typically have a long duration of time. Nevertheless, understanding whether these two classes of cells are differently affected by AD pathologies such as $\mathrm{A} \beta$ accumulation will be of great clinical importance.

Early reports showed place cell dysfunctions in $\mathrm{AD}$ model mice such as declined intersession stability, disrupted remapping, and expanded field size (Zhao et al., 2014; Mably et al., 2017; Jun et al., 2020). However, these studies have several limitations. First, the results were obtained by using tetrode recording in acute experiments, which is significantly limited in duration compared with the progression of the disease. The number of observed cells was limited. Also, there is no spatial information in relation to the pathologic changes. In contrast, $\mathrm{AD}$ pathology develops over decades even before the typical clinical or behavioral symptom manifests itself (Sasaguri et al., 2017). Early changes might be subtle and only occur in a small subset of neurons near the initial site of deposition or future sites of the deposition. Previous studies have focused on changes occurring at specific time points between control and $\mathrm{AD}$ model mice; however, longitudinal monitoring of large populations of neurons have the potential to be more informative and better identify long-term changes in activity and how these develop over time in relation to the pathologic changes (Berkowitz et al., 2018; McQuail et al., 2021). Likewise, such techniques are a prerequisite for identifying changes in neuronal properties occurring across distributed populations of neurons, such as synchronous firing, which cannot be detected unless a sufficiently large number of neurons are monitored simultaneously.

To capture a holistic view of the impact of $\mathrm{A} \beta$ pathology on the circuit activity, we used two-photon microscopy to visualize the activity of $\sim 1000$ neurons per animal and $\mathrm{A} \beta$ depositions in the dorsal hippocampal CA1 region of awake head-fixed knockin mice carrying humanized $A p p$ with Arctic, Swedish, and Beyreuther/Iberian mutations crossed with Thy1-G-CaMP7T2A-DsRed2 animals (Saito et al., 2014; Sato et al., 2020). Serendipitously, this animal allows for fluorescent detection of $\mathrm{A} \beta$ depositions in live, unstained brain tissue. By placing this animal in a virtual reality setup, we found that place cells were impaired in the hippocampus especially in the vicinity of $\mathrm{A} \beta$ depositions, whereas time cells were more resilient, suggesting distinct mechanisms for the formation of spatial- and temporal-encoding cells. This is the first longitudinal observation of hippocampal activity at the single-cell level simultaneous while monitoring A $\beta$ accumulation, providing versatile information to understand the pathogenesis of episodic memory in $\mathrm{AD}$ as well as revealing a therapeutic target or timing for this intractable disease.

\section{Materials and Methods}

Animals. Animal experiments were carried out in accordance with the institutional guidelines and protocols approved by RIKEN and Kyoto University. The animals were maintained on a $12 \mathrm{~h}$ light/ $12 \mathrm{~h}$ dark cycle. The behavioral experiments were performed in the dark phase. AD model mice were male single App knock-in that carry the Arctic, Swedish, and Beyreuther/Iberian mutations ( $A p p^{N L-G-F / N L-G-F}$; Saito et al., 2014). To observe the hippocampal CA1 neural circuit, the mice were crossed with transgenic mice coexpressing G-CaMP7 and DsRed2 via 2A peptide sequence from the Thosea asigna virus under the Thyl promoter
(Thy1-G-CaMP7-T2A-DsRed2 mice; Manita et al., 2015; Sato et al., 2015). For simplicity, Thy1-G-CaMP7-T2A-DsRed $2^{+1-}$ mice are shown as wildtype (WT)-G-CaMP7 mice, and $A p p^{N L-G-F / N L-G-F} /$ Thy1-G-CaMP7-T2ADsRed $2^{+/-}$mice are shown as AD-G-CaMP7 mice in this article.

Surgery. The surgery was performed in 2.5-month-old mice. Under anesthesia with $1.5 \%$ isoflurane, three anchor screws were inserted into the skull, and a stainless head plate $(25 \mathrm{~mm}$ length, $4 \mathrm{~mm}$ width, $1 \mathrm{~mm}$ thickness) with a circular opening $(7 \mathrm{~mm}$ inner diameter and $10 \mathrm{~mm}$ outer diameter; Sato et al., 2020) was attached to the skull with dental cement (SHOFU). The center of the circular opening was set at $2.0 \mathrm{~mm}$ posterior and $2.0 \mathrm{~mm}$ lateral to the bregma over the left hippocampus. Several days after the attachment of the head plate, a $2.5 \mathrm{~mm}$-diameter circular craniotomy was performed at the center of the opening of the head plate. The bare minimum of cortex was removed to expose the callosal surface above the hippocampus by aspiration, and an imaging window was implanted. The imaging window consisted of a polycarbonate ring $(2.5 \mathrm{~mm}$ outer diameter, $2.0 \mathrm{~mm}$ inner diameter, and $1.0 \mathrm{~mm}$ height) with a round coverslip $(2.5 \mathrm{~mm}$ diameter, $0.17 \mathrm{~mm}$ thickness; Matsunami Glass) attached at the bottom. To decrease the brain movement, a cohesive silicone sheeting $(0.005 \mathrm{~mm}$ thickness; Specialty Manufacturing) was attached to the bottom of coverslip. No noticeable differences of task performances, place cell properties, and learning ability were observed after the surgery (Dombeck et al., 2010; Sakaguchi et al., 2012). After recovery for 1 month, the mice were handled by a trainer for $10 \mathrm{~min}$ and then habituated to a Styrofoam cylinder treadmill (width $10 \mathrm{~cm}$ and diameter $20 \mathrm{~cm}$ ) for $10-30$ min per day, which was repeated for $5 \mathrm{~d}$. The behavioral training began after $3 \mathrm{~d}$ of water restriction. All mice were kept at $\sim 80 \%$ of presurgery body weight.

Virtual reality setup. The virtual reality (VR) setup consists of a Styrofoam cylinder treadmill and an interactive VR projection system (Sato et al., 2017). A single wide LCD monitor displayed VR graphics. The LCD monitor was placed $30 \mathrm{~cm}$ in front of the mouse on the treadmill. This covers an $81^{\circ}$ horizontal field of view and a $51^{\circ}$ vertical field of view. Rotation of the treadmill was detected by a USB optical detector (G400, Logitech). The VR paradigm consists of two components, a fixed-distance task and a fixed-time task, alternated without interval. In the fixed-distance task, a computer-generated virtual landscape (OmegaSpace 3.1, Solidray) as viewed from the mouse's virtual position was interactively displayed on the LCD monitor. The virtual linear track $(120 \mathrm{~cm}, 60$ bins) consists of wall $(4 \mathrm{~cm}$ high) and floor $(9 \mathrm{~cm}$ wide) with black-and-white patterns. Three large objects were placed outside the track as distal cues (a building, a mountain, and a tower). Three zones $(120 \mathrm{~cm}$ in length, at $30,60,90 \mathrm{~cm}$ from the start of the track) were marked with different wall patterns and color and served as visual cues for reward delivery points. In the fixed-time task, a still scene of the end wall is shown for $3 \mathrm{~s}$, which automatically switched to the scene of the start of the track, shown for $1 \mathrm{~s}$. During this period, the mice are still allowed to run on the trackball, but the movement is not reflected in the VR.

Behavioral tasks. The mice were head fixed on the cylinder treadmill. A waterspout connected to a pump (O'Hara \& Co.) was positioned within the reach of their tongue. The mice began the fixed-distance task by running from one end of the virtual linear track to seek water rewards delivered at one of the three target zones. Mice were rewarded immediately when they entered the reward zone. The reward was located at the middle target zones (green) in sessions \#1-15 (\#1-5, \#6-10, and \#11-15: early, middle, and late periods, respectively) and at the distal target zone (blue) in sessions \#16-20 (relearning period). Mice were water restricted for $3 \mathrm{~d}$ before starting tasks. Mice ran from the start point of the virtual linear track and received $5 \mu \mathrm{l}$ water three times, delivered every second, as a reward when entering green area. On reaching the end wall, the mice moved to the fixed-time task. VR was stopped for $3 \mathrm{~s}$, after which the start scene of the track was shown for $1 \mathrm{~s}$. The mice were then allowed to resume the fixed-distance tasks again. Mice performed these tasks at 4 and 7 months old. One session lasted $10 \mathrm{~min}$, and two sessions were conducted on each day with at least $3 \mathrm{~h}$ interval. A total 20 sessions were conducted in $10 \mathrm{~d}$. Behavioral data were collected at $50 \mathrm{~Hz}$ by custom software written in LabView (National Instruments). The sessions were removed from further analyses if a mouse did not run $>1$ lap $(120 \mathrm{~cm})$. 
A

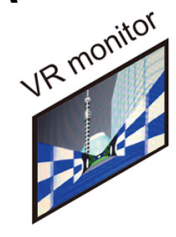

B

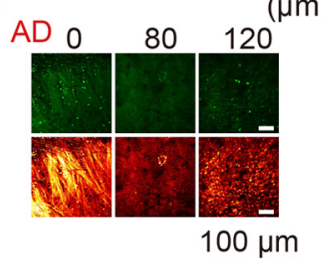

D

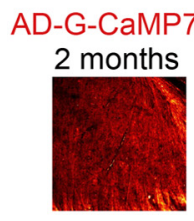

$2 p$ calcium imaging

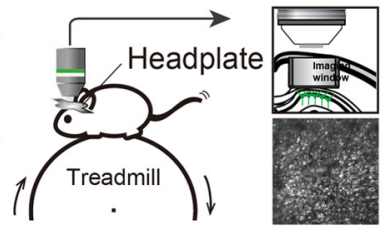

Fixed distance task

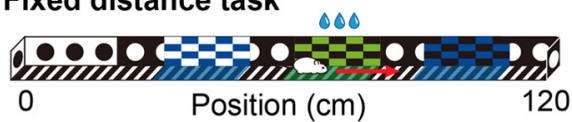

Fixed time task

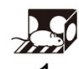

1

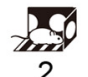

C
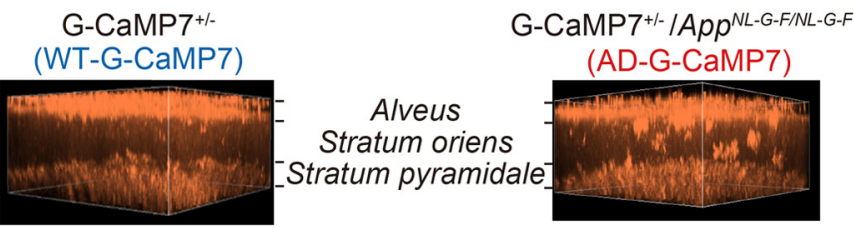

E

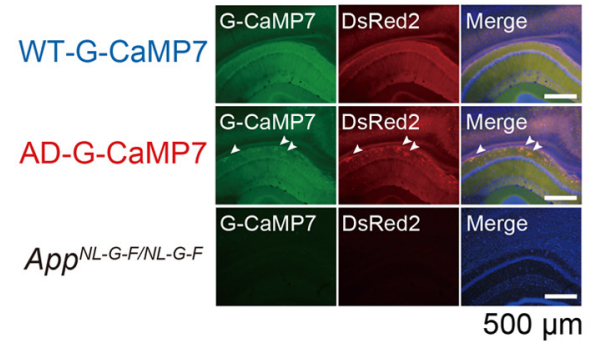

Figure 1. Emergence of G-CaMP7- and DsRed2-positive aggregates in AD-G-CaMP7 mice in vivo. A, Virtual navigation task and two-photon microscopic calcium imaging of hippocampal neuronal activity. The mice alternated between a fixed-distance task and a fixed-time task. During the fixed-distance task, the mice freely ran through a track with three areas marked with checkerboard patterns and different colors. The water reward was given at one of the areas. During the fixed-time task, the mouse is shown a fixed image of the end wall for $3 \mathrm{~s}$ and the start location of the track for $1 \mathrm{~s}$. During this period, the mice were allowed to run on the trackball, but the movement was not reflected in the virtual reality (VR). These two tasks were alternated without interval. B, In vivo two-photon microscopic images at different depths (left, alveus; middle, stratum oriens; right, stratum pyramidale) in a 4-month-old AD-G-CaMP7 mouse, implanted with an imaging window above the hippocampal dorsal CA1 region. Numbers show depth from alveus. Most of G-CaMP7- and DsRed2-positive (G7D2) aggregates were observed at stratum oriens. C, A side view of three-dimensional reconstructions $(532 \mu \mathrm{m} \times 532 \mu \mathrm{m} \times 170 \mu \mathrm{m}$ ) of WT-G-CaMP7 and AD-G-CaMP7 mice both at 4 months old. Note the presence of aggregates in the stratum oriens from the AD-G-CaMP7 image, but they are largely absent in the WT-G-CaMP7. D, Appearance of $A \beta$ aggregates at stratum oriens of hippocampal CA1 region in AD-G-CaMP7 mouse. A $\beta$ aggregates were not observed in 2-month-old AD-G-CaMP7 mouse but appeared at 2.5 months. $E$, Fluorescent images of hippocampal sections of WT-G-CaMP7 (left), AD-G-CaMP7 (middle), and App ${ }^{N-G F N L-G F}$ mice (right) at 14 months old. The sections were counterstained with Hoechst 33258.

Data acquisition. Calcium imaging during awake behavior was performed using a two-photon microscope (Nikon A1 MP) equipped with a $16 \times$ NA 0.8 objective and external GaAsP photomultiplier tubes. A Tisapphire laser (Mai Tai eHP DeepSee, Spectra-Physics) tuned at $910 \mathrm{~nm}$ was used to excite G-CaMP7 and DsRed2. The signal was separated by a $570 \mathrm{~nm}$ dichroic mirror, a $495-540 \mathrm{~nm}$ bandpass filter for G-CaMP7, and a $575-630 \mathrm{~nm}$ bandpass filter for DsRed2. Images of $512 \times 512$ pixels $(\sim 532 \mu \mathrm{m} \times 532 \mu \mathrm{m})$ were acquired at $15 \mathrm{~Hz}$ using a resonant-galvo scanner.

Detections of $\mathrm{Ca}^{2+}$ activity. The imaging movie was acquired using NIS-Elements software at $15 \mathrm{~Hz}$ (Nikon), converted to TIF files, and aligned to reduce misalignment caused by brained movement using the TurboReg ImageJ plugin. A maximum intensity image was obtained from the movie. After a median image of all frames was subtracted from each image of the aligned images, the noise was removed by applying a median filter $(5 \times 5$ pixels). Then, image maximum intensity was calculated from a stack of the denoised image. The imaging movie was smoothed by a spatio-temporal median filter $(7 \times 7 \times 3$ pixels cuboid neighborhood), and the footprint with spatial weight of each cell in the movie was identified by a modified non-negative matrix factorization algorithm (Takekawa et al., 2017). To avoid interference with neighboring cell footprints, a footprint $<40 \mathrm{SD}$ in the whole imaging area was removed. If two footprints had overlapping areas larger than $20 \%$, the smaller one was removed. Using the remaining footprints, the calcium trace of each cell was calculated from the movie and was smoothed by the low-pass filter and the Savitzky-Golay smoothing filter. The basal level of calcium trace was determined from the mode and set to zero. Calcium intensities higher than an arbitrary threshold ( 0.05 , approximately equal to $3 \mathrm{SD}$ of calcium signals) in the rising phase of fluorescence transient were detected as activity, and the event rate (\% of total measurement time) was obtained from active frames divided by the total number of recording frames for each cell. We here define a cell as hyperactive if it has event rate of $>10 \%$, which roughly corresponds to the top 95th percentile value (10.68\%) in 4-month-old WT-G-CaMP7 mice (see Fig. $3 A, B)$. The same value was used in all subsequent sessions and all ages. The total recorded frames, from both fixed-distance and fixed-time tasks, were classified into run and stop phases. Run and stop phases were calculated by running and stopping frames on the treadmill. The obtained footprints were used to calculate the cell activity in all sessions.

To identify the same cell over multiple sessions, the new footprints were constructed by comparing footprints obtained in the first 10 sessions from 4-month-old mice. When more than half the area of one footprint in a session overlapped with one footprint in another session, and the distance between their centroids was $<5$ pixels, the larger of the two footprints remained. If a footprint was matched over three times in 10 sessions, it remained, and these footprints were used for constructing multiple footprints. In other sessions, the footprint of a cell that was matched to one of the constructed multiple footprints was used for the analysis to trace the same cell over months (see Figs. 5D,E, 11D,E).

Analysis of virtual place cells. Data from the fixed-distance task were used to detect the virtual place cells. Two analyses, regression analysis (Miri et al., 2011) and mutual information (MI) analysis (Ziv et al., 2013; Sato et al., 2020), were used. Regression analysis was conducted as follows:

$$
Y=\beta_{0}+\beta X
$$

where $Y$ is a measured variable, $\beta$ is the weight matrix of regression expressing how the weighted sum of another variable $X$ can reconstruct $Y$, and $\beta_{0}$ is the population $Y$ intercept. The term is considered as the offset value of the linear regression. If we hypothesized that the place 
cells fired because of a visit by the animals at a specific location, we can use the linear regression method as described above to find the weights of each place cell at spatial bins. First, we divided the linear track length in 60 bins (one bin $=10$ units) and formed a matrix of place vectors. Each session had 8,989 imaging frames for $10 \mathrm{~min}$, so the dimension of place vectors was $60 \times 8,989$. For each bin, if the position of a cell on the linear track at a specific frame was inside that bin, a value of one was assigned. Otherwise zero was assigned. These place vectors formed a matrix of Values 1 and 0 and was assigned as $\mathrm{X}$ in the above equation. We calculated the spike matrix of all detected cells and assigned this matrix (number of cells by 8,989 frames) as $\mathrm{Y}$ in the above equation. By doing this, we wanted to calculate $\beta$, the regression weights, with a dimension of (number of cells by 60 bins). The resultant weight matrix $\beta$ showed how strongly each cell encoded each bin. After obtaining the weight matrix through regression, the weight matrix was $Z$-scored, with an intention of normalization over sessions and animals. Then for each bin, we looked for cells with a higher value of weight. To do this, we picked cells that had a weight as large as three or more (corresponding to 3 SDs of the weight matrix data before $Z$-score), for each bin. With this method, we found several cells for each spatial bin that encoded that specific bin much stronger than other cells. We assigned these cells as virtual place cells for that bin. Then we gathered such virtual place cells for all 60 bins and labeled them together as virtual place cells of that session. Next, we calculated the MI and the peak of mean calcium activity rate of each virtual place cell in a mouse's virtual linear track position (Ziv et al., 2013; Sato et al., 2020). The mean calcium activity rate was calculated for each bin in a session and smoothed by a Gaussian filter (Gaussian window size $=3$ frames, $\sigma=1.4$ ). We defined the smoothed average event frequency as a place field. We also calculated MI and the maximum values of place fields using data that were obtained by randomly rotating the activity during running on each trial. This process repeated 1000 times for each cell. If MI and the maximum value of place field in real data were $>95$ th percentile of values obtained from random permutation data, cells were considered as virtual place cells. Therefore, virtual place cells were identified from regression and MI analyses. Also, the cell was removed from place cell analyses if the activity was $>30 \%$ trials in a session.

Similarity between the place field of each cell in consecutive sessions A (\#n) and B $(\# n+1)$ was analyzed by the Pearson correlation coefficient $(\rho)$, as follows:

$$
\rho(\mathrm{A}, \mathrm{B})=\frac{1}{N-1} \sum_{i}\left(\frac{A_{i}-\mu_{A}}{\sigma_{A}}\right)\left(\frac{B_{i}-\mu_{B}}{\sigma_{B}}\right),
$$

where $N$ is the number of all bins, $i$ is the bin number, $A_{i}$ is the firing rate at bin $\# i$ in session $\# n, \mu_{A}$ is the average of firing rates, and $\sigma_{A}$ is the $\mathrm{SD}$ of firing rates. If similarity and peak shift of place field in a virtual place cell between two consecutive sessions were over 0.5 and less than $\pm 8 \mathrm{~cm}$, respectively, it was designated as stable place cell.

Place field size for each cell was calculated by counting the total number of bins with more than half of the maximum value in the place field.

Analysis of time cells. The data from the fixed-time task were used to analyze the time cell activity. Time-locked activity was calculated during
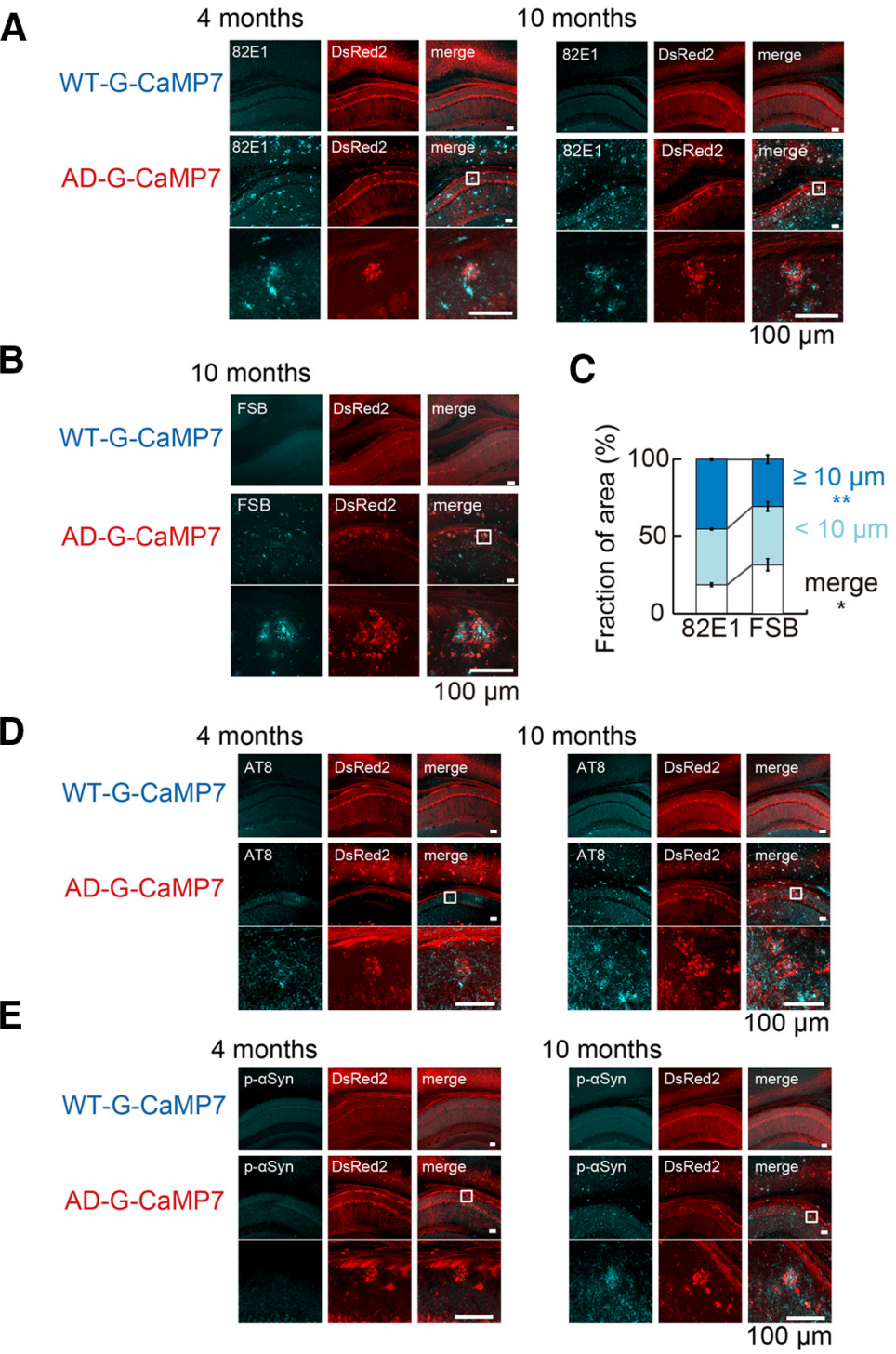

Figure 2. G7D2 aggregations are closely linked with aggregated $A \beta$. $A$, Tissue staining of hippocampal CA1 regions of 4-month-old (left) and 10-month-old (right) AD-G-CaMP7 mice with anti-human A $\beta$ antibody (82E1). Images in botregions of 10-month-old WT-G-CaMP7 mice with 1-fluoro-2,5-bis (3-carboxy-4-hydroxystyryl) benzene. C, Shortest disbetween $A \beta$ signals and area of $A \beta$-DsRed2 overlap was quantified for each pixel at 10 months $(n=3)$. MP7 and AD-G-CaMP7 mice at 4 and 10 months with anti-phospho-PHF-tau pSer202+Thr205 (AT8, D) and anti-phosphorylated $\alpha$-synuclein (p- $\alpha$ Syn, $\boldsymbol{E}$ ).

the $3 \mathrm{~s}$ at the end of a lap and $1 \mathrm{~s}$ more at the beginning of the next lap, where the mice were typically still running on the cylinder, plus $2 \mathrm{~s}$ before and after (see Fig. 6A). The resultant time field was smoothed with Gaussian function (Gaussian window size = five frames, $\sigma=2.5$ ). To judge the significant time-locked activity, we compared the maximum value of a time field to that of 1000 random data permutations in the end period of each lap for the same cell. The random permutation was conducted in each lap. If the maximum value of time field in real data was greater than the 95 th percentile of the value obtained from the permutated data, the cell was defined as time cell.

In addition to place cell stability analysis as mentioned above, a time cell was identified as a stable time cell if the similarity of the time field calculated by the Pearson correlation coefficient, was $>0.5$, and a peak shift of time field was less than \pm 4 frames $(=66.7 \mathrm{~ms})$ between two consecutive sessions.

Analysis of size and density of G-CaMP7 and DsRed2-positive aggregates. The size of G-CaMP7 and DsRed2-positive (G7D2) aggregates 

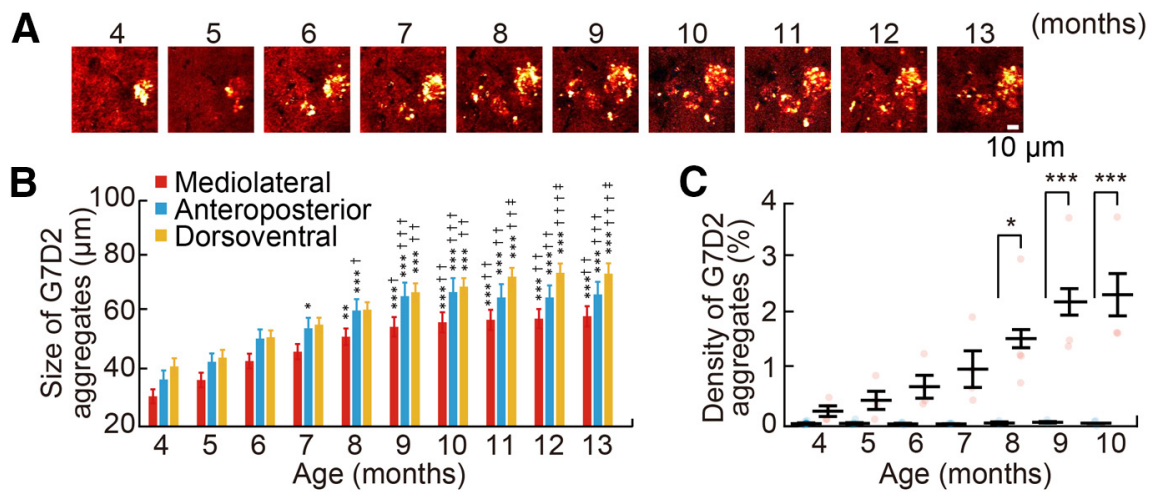

C

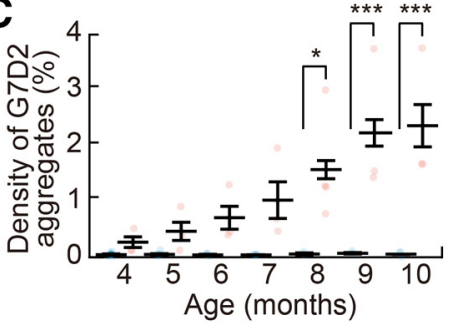

D
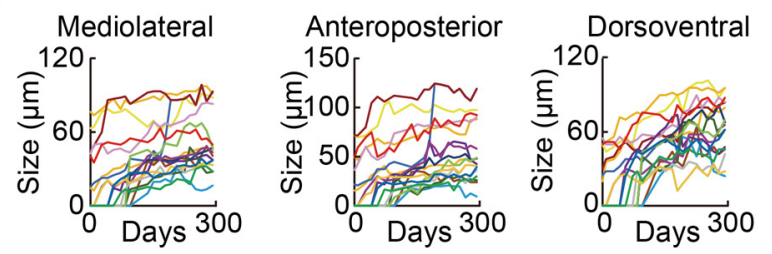

Figure 3. G7D2 aggregations expand with age in AD-G-CaMP7 mice. $A$, Progression of G7D2 aggregations in stratum oriens in AD-G-CaMP7 mouse over 9 months. $B$, Growth of each G7D2 aggregation from 4 to 13 months; $n=46,55,49,61,64,56$, $55,43,43,43$ aggregations in each month; $p=2.96 \times 10^{-44}$ by two-way ANOVA effect of age, $F_{(9,1405)}=27.53$. Two-way ANOVA followed by a Tukey-Kramer post hoc test ( ${ }^{*}$ versus $4 \mathrm{~m}$, tversus $5 \mathrm{~m}$, fversus $6 \mathrm{~m}$; ${ }^{*} p<0.05$, ${ }^{* *} p<0.01$, ${ }^{* * *} p<$ 0.001). C, Occupancy of G7D2 aggregations in the stratum oriens in $30 \mu \mathrm{m}$ thick and $532 \mu \mathrm{m}$ square stratum oriens in AD-GCaMP7 $(n=3)$ and WT-G-CaMP7 mice $(n=3) ; p=2.38 \times 10^{-7}$ by two-way ANOVA effect of genotype, $F_{(1,27)}=46.8$. Twoway ANOVA followed by a Tukey-Kramer post hoc test $\left({ }^{*} p<0.05\right.$, $\left.{ }^{* * *} p<0.001\right)$. $\boldsymbol{D}$, The growth of $A \beta$ aggregates from 4 to 14 months. Each color represents each aggregate.

was measured in the direction of mediolateral, anteroposterior, and dorsoventral manually by using NIS-Elements software. The density of G7D2 aggregates was analyzed by counting pixels with a fluorescent intensity beyond threshold. The original image was processed with MATLAB; unevenness of background was corrected and subtracted from the original image. Then we calculated the density of aggregates whose signals were more than the determined threshold. The threshold $(S)$ was set as follows:

$$
S=M+3 H_{W T},
$$

where $M$ is the mode, the most frequent intensity, of each image, $H_{W T}$ is the half peak duration of the intensity histogram in WT-G-CaMP7. The half-width of the intensity histogram is different between WT-G-CaMP7 and $\mathrm{AD}-\mathrm{G}-\mathrm{CaMP} 7$ because of high intensity aggregates, so the intensity histogram of WT-G-CaMP7 was used.

Analysis of distance to G7D2 aggregates. To examine the relationships between G7D2 aggregates and cells, the shortest distance from each cell to the center of the nearest G7D2 aggregates was measured for each cell. The center of the aggregate is calculated by the schematic ellipse, which was generated from the position and size of aggregates.

Immunostaining. Mice were anesthetized by intraperitoneal injection of avertin and perfused intracardially with $4 \%$ paraformaldehyde (PFA) in PBS. Brains were further fixed with $4 \%$ PFA in PBS at $4^{\circ} \mathrm{C}$ overnight. Coronal sections were made at $60 \mu \mathrm{m}$ thickness on a vibratome. For immunostaining, antibodies to the $\mathrm{N}$-terminal region (1-16) of $\mathrm{A} \beta$ (82E1; 1:1000; catalog \#10323, IBL International), Ser202- and Thr205-phosphorylated-paired helical filament (PHF)-tau (AT8; 1:1000; catalog \#MN1020, Invitrogen), and Ser129-phosphorylated $\alpha$-Synuclein (p- $\alpha$-Syn; 1:1000; catalog \#015-2591, Wako) were used. To detect $\beta$-sheet structure of A $\beta$ (Sato et al., 2004), 1-Fluoro-2,5-bis (3-carboxy-4-hydroxystyryl) benzene (FSB; 1:1000; catalog \#34190811, Dojindo) was used. Hoechst 33258 (1:2000; catalog \#382061, Calbiochem) was used for the nuclear staining. Images were obtained by laser scanning confocal microscopes (FluoView FV1000 microscope, Olympus, or BZ-X700 fluorescence microscope, KEYENCE).
For each color image, the intensity higher than threshold (2 SDs of selected pixels in CA1 region) were detected as signals. By comparing images of $\mathrm{A} \beta$ and DsRed2, the pixel-by-pixel distances of the $\mathrm{A} \beta$ signals from the nearest overlapping pixel (shortest distance from each signal pixel to overlap pixels $<10 \mu \mathrm{m}$ ) between $\mathrm{A} \beta$ and DsRed 2 were calculated.

Statistics. When only two groups were compared, Student's $t$ tests or Wilcoxon rank sum tests were used. When more than two groups were compared, ANOVA was used, and $p$ values were adjusted for post hoc multiple comparisons. Bar and dot plots are represented with mean \pm SEM. Violin plots are represented with mean (black solid line) and median (red dotted line). Each dot represents the value of a session. Box plots are represented with mean (circle) and median (midline).

\section{Results}

\section{G-CaMP7 and DsRed2 form fluorescent aggregates overlapping with $\mathrm{A} \beta$ deposition}

Throughout the study, we used a hybrid between $A p p^{N L-G-F / N L-G-F}$ knock-in mice and Thy1-G-CaMP7-T2A-DsRed2 transgenic mice (hereafter described as $\mathrm{AD}$ G-CaMP7). The $A p p^{N L-G-F / N L-G-F}$ knockin mouse model enabled us to explore the impacts of $\mathrm{A} \beta$ pathology without destroying endogenous gene loci or overexpression, unlike other AD model mouse lines (Saito et al., 2014; Sasaguri et al., 2017). This model exhibits progressive $\mathrm{A} \beta$ pathology, but not tau pathology or brain atrophy, which indicates we can approach the neuronal dysfunctions under $\mathrm{A} \beta$ pathogenesis. On the other hand, Thy1-G-CaMP7-T2A-DsRed2 transgenic mice coexpresses G-CaMP7 and DsRed2 proteins under Thy1 promotor (Sato et al., 2020). G-CaMP7 is a $\mathrm{Ca}^{2+}$-sensitive fluorescent protein that allows us to detect the neuronal activity in the form of calcium transients (Ohkura et al., 2012). DsRed2 is a red fluorescent protein that shows stable fluorescence regardless of the neuronal activity. Here, we used it to precisely align the imaging field across days. We imaged the CA1 region of the dorsal hippocampus of AD-G-CaMP7 mice through an imaging window implanted in the overlying cortex using a two-photon microscope (Fig. 1A).

In addition to expected dynamic G-CaMP7 signals reflecting neuronal activity and static DsRed2 signals normally seen in the stratum pyramidale of Thy1-G-CaMP7-T2ADsRed $2^{+/-}$(WT-G-CaMP7) mice, we found unexpected static green- and red-fluorescent (G-CaMP7- and DsRed2positive fluorescent: G7D2) aggregates in the stratum oriens of AD-G-CaMP7 mice (Fig. $1 B, C$ ). We did not detect aggregates in strata pyramidale and radiatum. Such aggregates were not seen in mice carrying either Thy1-G-CaMP7-T2ADsRed $2^{+/-}$or $A p p^{N L-G-F / N L-G-F}$ locus only. The onset of the aggregation was $\sim 2-2.5$ months of age, consistent with the appearance of $\mathrm{A} \beta$ in $A p p^{N L-G-F / N L-G-F}$ mice (Saito et al., 2014; Fig. $1 D$ ). Therefore, it is likely that the fluorescence aggregates represent $\mathrm{A} \beta$ depositions that take up G-CaMP7 and DsRed 2 proteins. Consistent with in vivo imaging, the green and red aggregates in brain sections were observed in 

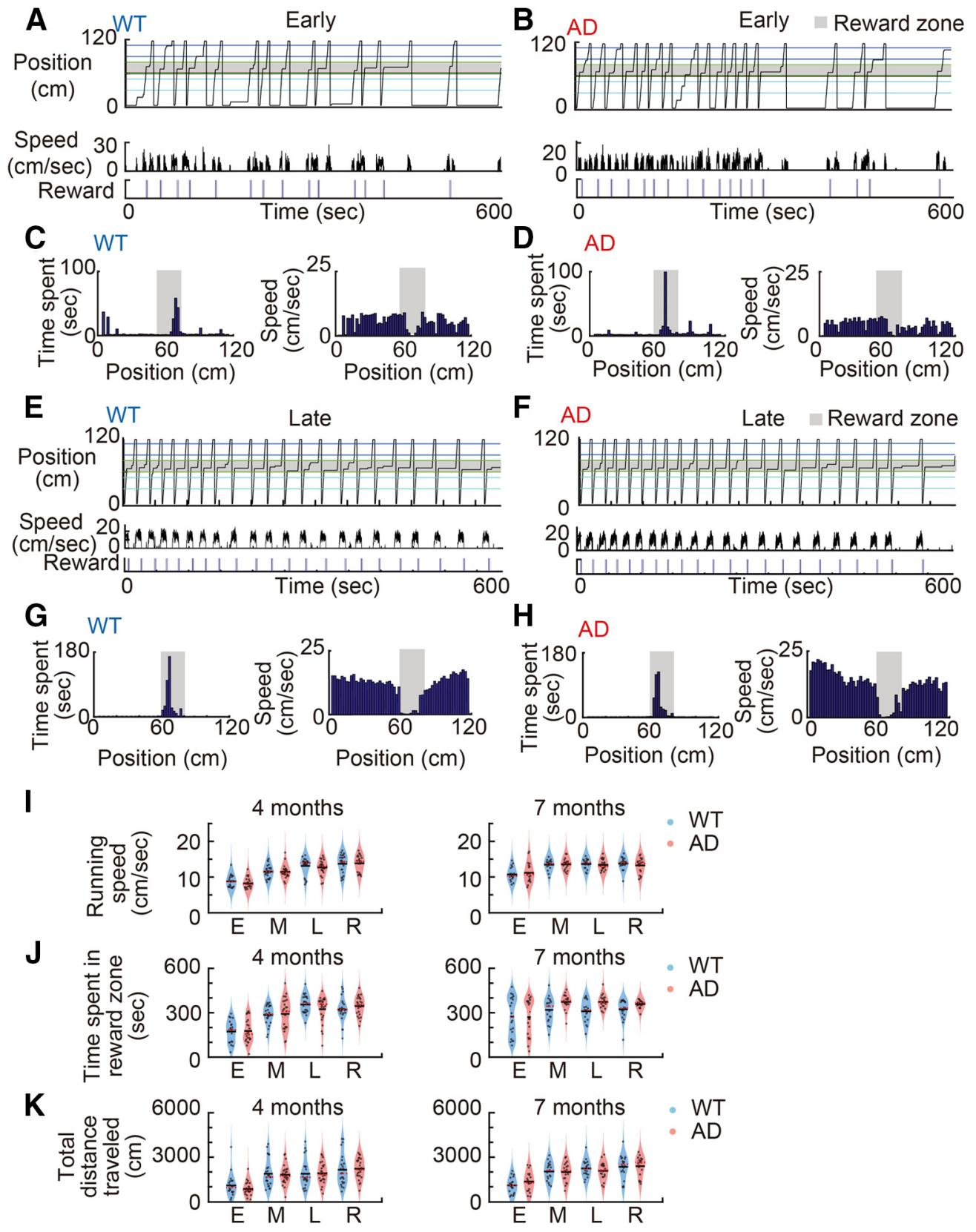

Figure 4. Behavior in VR is not largely affected in AD-G-CaMP7 mice. $\boldsymbol{A}-\boldsymbol{H}$, Examples of behavior of 4-month-old WT-G-CaMP7 $(\boldsymbol{A}, \boldsymbol{E})$ and AD-G-CaMP7 $(\boldsymbol{B}, \boldsymbol{F})$ mice at early $(\boldsymbol{A}, \boldsymbol{B})$ and late $(\boldsymbol{E}, \boldsymbol{F})$ periods. The time spent at each location and speed are shown $(\boldsymbol{C}, \boldsymbol{D}, \boldsymbol{G}, \boldsymbol{H})$. $\boldsymbol{I}-\boldsymbol{K}$, Violin plots of running speed $(\boldsymbol{I})$, time in reward zone $(\boldsymbol{J})$, and total distance $(\boldsymbol{K})$ in early $(\boldsymbol{E})$, middle $(\boldsymbol{M})$, late $(\boldsymbol{L})$, and relearning $(\boldsymbol{R})$ periods of 4 - and 7-month-old WT-G-CaMP7 and AD-G-CaMP7 mice. Four months old: WT = five mice (20-25 sessions), AD = five mice (24-25 sessions). Seven months old: WT $=$ four mice (18-20 sessions), $A D=$ four mice (20 sessions). $I$, Four months old: $p=0.43$ by two-way ANOVA effect of genotype, $F_{(1,186)}=0.62$. Seven months old: $p=$ 0.82 by two-way ANOVA effect of genotype, $F_{(1,149)}=0.05$. J, Four months old: $p=0.94$ by two-way ANOVA effect of genotype, $F_{(1,186)}=0.0066$. Seven months old: $p=0.0057$ by two-way ANOVA effect of genotype, $F_{(1,149)}=7.88$. $K$, Four months old: $p=0.63$ by two-way ANOVA effect of genotype, $F_{(1,186)}=0.23$. Seven months old: $p=0.78$ by two-way ANOVA effect of genotype, $F_{(1,149)}=0.08$.

the stratum oriens of AD-G-CaMP7 but not of WT-GCaMP7 mice (Fig. 1E).

The aggregates reacted with two $\mathrm{A} \beta$ markers-82E1, which detects the $\mathrm{N}$ terminus of $\mathrm{A} \beta$, and FSB, which detects the $\beta$-pleated sheet structure (Fig. $2 A, B$ ). The signals for FSB were mainly detected in stratum oriens and much sparser in stratum radiatum, whereas those for 82E1 were detected in both. G7D2 aggregates more closely colocalized with the staining with FSB than $82 \mathrm{E} 1$, indicating that the aggregates represent highly aggregated $\mathrm{A} \beta$ (Fig. $2 \mathrm{C}$ ). We also stained the section with antibodies for phosphorylated tau (AT8), the main component of neurofibrillary tangles (Fig. 2D), and for Ser129-phosphorylated $\alpha$-synuclein (p- $\alpha$ Syn; Fig. 2E; Kaneko et al., 2007; Muntané et al., 2008). Signals for AT8 and p- $\alpha$ Syn were seldom colocalized with aggregates in 4-month-old tissue, but at 10 months, both signals colocalized with the aggregates, indicating that these pathogenic proteins are gradually entangled in the aggregates.

We chronically imaged the same visual field over nine months. The number, size, and density of the G7D2 aggregates increased in all three dimensions with age (Fig. $3 A-D$ ). These results suggest that the fluorescent aggregates seen in $\mathrm{AD}$ G-CaMP7 mice represent $\mathrm{A} \beta$ depositions. We therefore used 
A
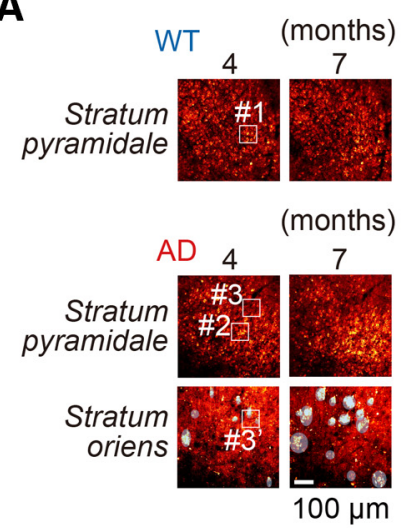

B
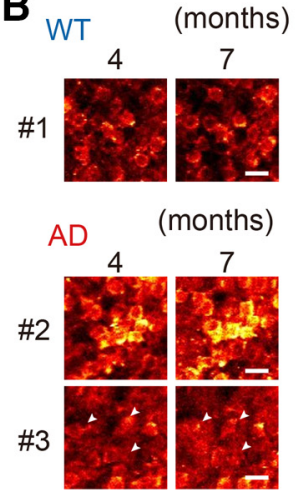

AD

\#3+ellipses

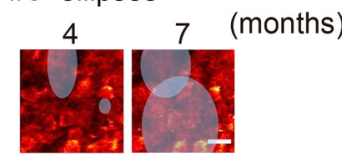

\#3'+ellipses

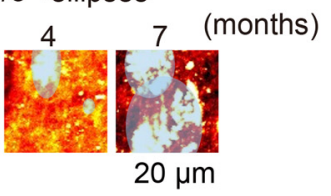

C
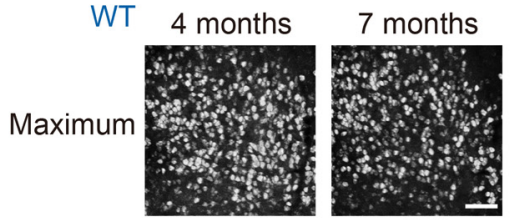

$100 \mu \mathrm{m}$

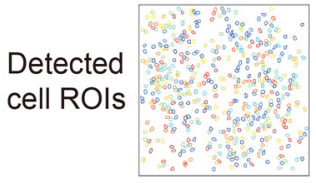

D

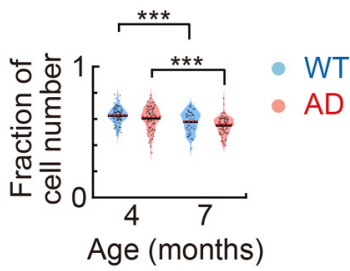

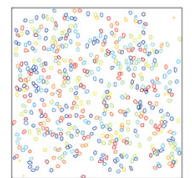

$E$

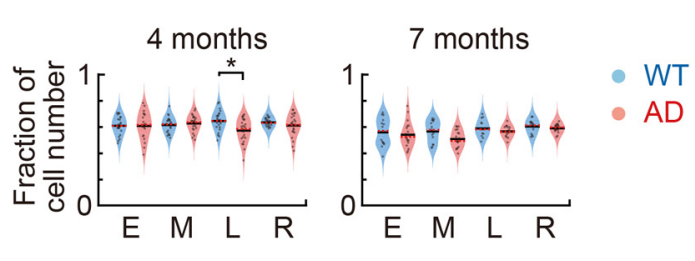

Figure 5. Chronic two-photon calcium imaging of the same pyramidal cell population. $A$, DsRed2 fluorescence images of strata pyramidale and oriens in 4- and 7-month-old WT-G-CaMP7 and AD-G-CaMP7 mice. B, Magnification of white rectangles (\#1, \#2, $\left.\# 3, \# 3^{\prime}\right)$ in $\boldsymbol{A}$. The same cell population was traced over three months in WT-G-CaMP7 and AD-G-CaMP7 mice even near a G7D2 aggregate. C, Maximum intensity projection images (top) and ROls detected by non-negative matrix factorization method (bottom) in 4- and 7-month-old WT-G-CaMP7 and AD-G-CaMP7 mice. D, Violin plots of the fraction of active cell numbers in 4- and 7-month-old WT-G-CaMP7 and AD-G-CaMP7 mice. Violin plots are represented with mean (black solid line) and median (red dotted line). Four months old: $W T=$ five mice ( 99 sessions), $A D=$ five mice (95 sessions). Seven months old: WT $=3$ mice (60 sessions), $A D=$ four mice (80 sessions); $p=0.0024$ by two-way ANOVA effect of genotype, $F_{(1,330)}=9.36, p=1.87 \times 10^{-9}$ by two-way ANOVA effect of age, $F_{(1,330)}=38.21$. $\boldsymbol{E}$, Breakdown of $\boldsymbol{D}$ to early $(\mathrm{E})$, middle $(\mathrm{M})$, late $(\mathrm{L})$, and relearning $(\mathrm{R})$ periods of 4- and 7-month-old WT-G-CaMP7 and AD-G-CaMP7 mice. Four months old: $p=0.043$ by two-way ANOVA effect of genotype, $F_{(1,186)}=4.15$. Seven months old: $p=0.0018$ by two-way ANOVA effect of genotype, $F_{(1,132)}=5.78$. Two-way ANOVA followed by a Tukey-Kramer post hoc test $\left({ }^{*} p<0.05,{ }^{* * *} p<0.001\right)$.

this animal to observe the disease progression in vivo while monitoring the neuronal activity.

\section{Both WT-G-CaMP7 and AD-G-CaMP7 mice learn to seek reward under virtual reality task}

We used a VR task that allows symmetric measure of spatial and temporal representations in the hippocampus by fixing either one of the parameters at a time while leaving the other ad libitum to the mice (Fig. 1A; Sato et al., 2017). In the fixed-distance task, the mouse freely runs through a segment of fixed length at its own speed to finish the lap. The visual cues are updated as the mice run for water rewards delivered at a fixed position on the track. In contrast, in the fixed-time task, the mice spend a fixed duration of $4 \mathrm{~s}$ with fixed visual cues. The mice can still freely run on the trackball, but the cues are not updated except that the scene switches from the end of the track to the start at $3 \mathrm{~s}$. The mice alternate between the fixed-distance and fixedtime tasks during a single session, which was conducted twice a day.

Both WT-G-CaMP7 and AD-GCaMP7 mice ran intermittently on the track in early sessions (\#1-5) of the training at 4 months of age. Then they started behaving in a more stereotypic manner by running faster and stopping only in the reward zone in the later sessions (\#6-15), even after the reward was shifted to another zone (\#16-20; Fig. 4A-H), consistent with our earlier study (Sato et al., 2020). There were no differences in running speed, time spent in the reward zone, and travel distance between the genotypes at 4 and 7 months (Fig. $4 I-K$ ), indicating the normal gross motor functions of AD-G-CaMP7 mice under $\mathrm{A} \beta$ pathology.

\section{Neurons in the vicinity of $A \beta$ aggregates became hyperactive in AD-G-CaMP7 mice with age}

We monitored the activity of dorsal hippocampal CA1 pyramidal neurons in the stratum pyramidale from 4 to 7 months of age (Fig. $5 A, B$ ). The number of active cells in each session ranged from 351-768 (median 558) in WT and from 355-791 (median 525) in $\mathrm{AD}$ mice. We then aligned images across different sessions and identified a total $925 \pm 51$ cells in WT and $936 \pm 53$ cells in $\mathrm{AD}$ mice. The G7D2 aggregates in the overlaying stratum oriens were approximated by ellipses and projected to the stratum pyramidale to detect cells beneath the aggregates. The neuronal activity while the mice are alternating the two tasks was detected by GCaMP7 imaging and was processed through a non-negative factorizationbased method (Takekawa et al., 2017; Sato et al., 2020; Fig. 5C). The fraction of active neurons in each session per detected neurons in the imaging field gradually declined with age in both genotypes but especially in AD-G-CaMP7 mice (Fig. $5 D, E$, age effect: $F_{(1,330)}=$ $38.21, p=0.0024$, genotype effect: $\left.F_{(1,330)}=9.36, p<0.001\right)$. This indicates that the number of active cells decreased with age during longitudinal $\mathrm{Ca}^{2+}$ imaging, especially in AD-G-CaMP7 mice.

Although there was a reduction in the fraction of active neurons in each session in AD-G-CaMP7 (Fig. 5D), overall behavior largely did not change with our task (Fig. 4). We wondered whether there is any compensatory mechanism in the remaining neurons. In 4-month-old animals, there was a significant increase of event rate (Fig. 6A). We defined a cell as hyperactive 


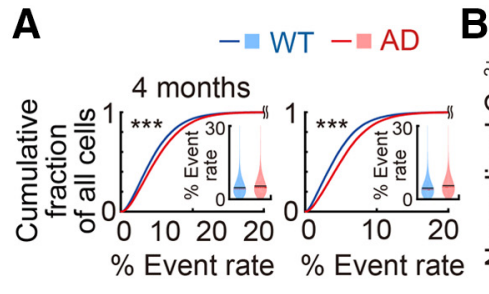

$\mathbf{D}_{7 \text { months }}$

Stratum pyramidale

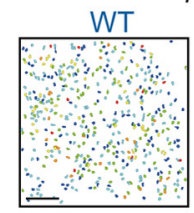

$100 \mu \mathrm{m}$

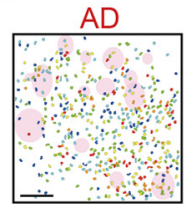

$B_{A D}$ Hyperactive

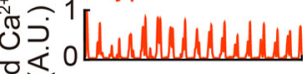
은 Non-Hyperactive
C

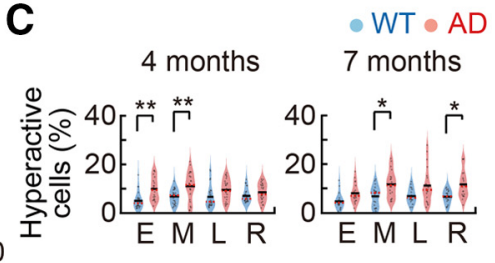

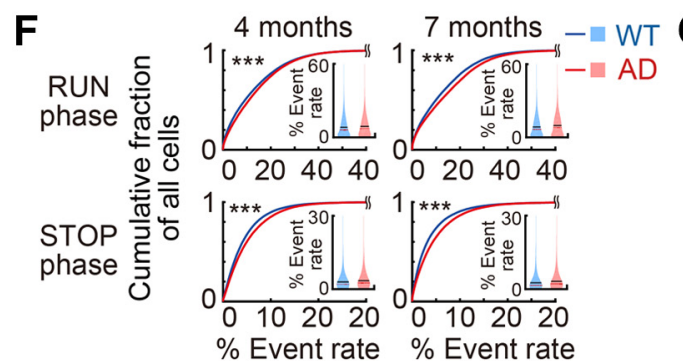

E

-n Non-hyperactive cells

- Hyperactive cells

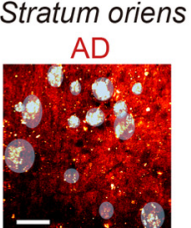

- $\geq 10 \%$

- $8-10 \%$

- $6-8 \%$

- $4-6 \%$

- $2-4 \%$

- $<2 \%$

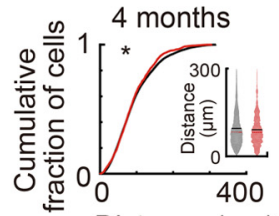

7 months

Distance $(\mu \mathrm{m})$

\author{
G
}

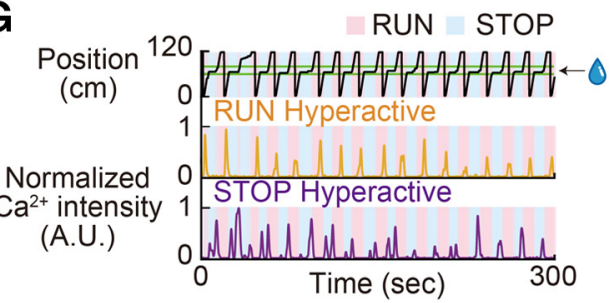

RUN phase

- WT $\bullet A D$

7 months

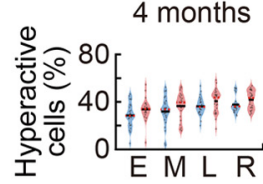

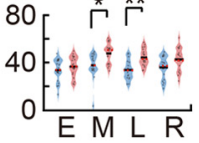

I STOP phase

4 months

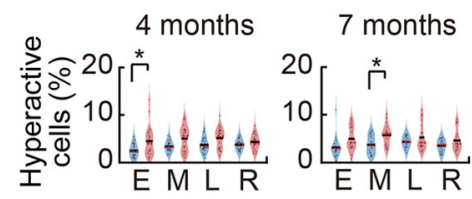

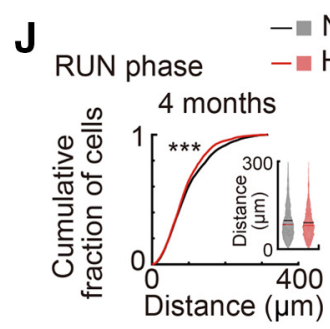

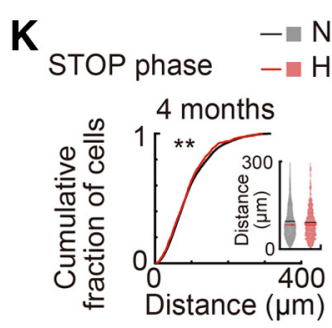

- Non-hyperactive cells

Non-hyperactive cells

Hyperactive cells

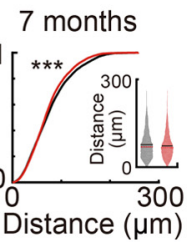

7 months

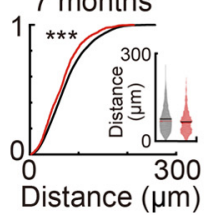

Figure 6. Increase of hyperactive cells near G7D2 aggregates in AD-G-CaMP7 mice. A, Cumulative histograms and violin plots of event rate of active neurons. Event rate of 4- and 7-month-old WT-GCaMP7 and AD-G-CaMP7 mice in total frames. Four months old: WT = five mice $\left(57,090\right.$ cells), $A D=$ five mice $\left(53,776\right.$ cells); $p=1.50 \times 10^{-237}, Z=-32.91$. Seven months old: $W T=$ four mice (42,032 cells), $A D=$ four mice $\left(40,076\right.$ cells), $p=0.00, Z=-44.10$. Wilcoxon rank sum test $\left({ }^{* * *} p<0.001\right)$. B, Examples of G-CaMP7 fluorescence of hyperactive (red) and nonhyperactive (blue) cells. Hyperactive cells were defined as those having activity in $\geq 10 \%$ of all frames. C, Violin plots of proportion of hyperactive cells among all active cells in early (E), middle (M), late (L), and relearning (R) periods during 10 min imaging in 4- and 7-month-old WT-G-CaMP7 and AD-G-CaMP7 mice. Four months old: WT $=$ five mice ( $20-25$ sessions), AD $=$ five mice $\left(24-25\right.$ sessions), $p=5.78 \times 10^{-8}$ by two-way ANOVA effect of genotype, $F_{(1,186)}=31.98$. Seven months old: $W T=$ four mice $\left(18-20\right.$ sessions), AD $=$ four mice $\left(20\right.$ sessions), $p=2.15 \times 10^{-8}$ by two-way ANOVA effect of genotype, $F_{(1,149)}=35.12$. Two-way ANOVA followed by a Tukey-Kramer post hoc test $\left({ }^{*} p<0.05,{ }^{* *} p<0.01\right)$. $\boldsymbol{D}$, Example of the maximum intensity projection image at the stratum oriens and the cell map of the stratum pyramidale with cellular activities indicated with red dots $(\geq 10 \%)$, orange dots (8-10\%), yellow dots $(6-8 \%)$, green dots $(4-6 \%)$, light blue dots $(2-4 \%)$, blue dots $(<2 \%)$, and $G 7 D 2$ aggregates with a pink ellipse from 7-month-old AD-G-CaMP7 mice. $E$, Cumulative histograms and violin plots of shortest distance from G7D2 aggregates to hyperactive cell (red) and nonhyperactive cells (black) in 4- and 7-month-old AD-G-CaMP7 mice. Four months old: $A D=$ four mice (hyperactive $=4165$ cells, nonhyperactive $=40494$ cells), $p=0.011, Z=-2.54$. Seven months old: $A D=$ four mice (hyperactive $=4,275$ cells, nonhyperactive $=35,801$ cells), $p=7.92 \times 10^{-14}, Z=-7.47$. Wilcoxon rank sum test ( $\left.{ }^{* * *} p<0.001\right)$. $F$, Cumulative histograms and violin plots of event rate of active neurons. Event rate of WT-G-CaMP7 and AD-G-CaMP7 mice in run frames (top) and stop frames (bottom). RUN 4 months old: $p=1.24 \times 10^{-86}, Z=-19.73 ; 7$ months old: $p=2.53 \times 10^{-86}, Z=$ -19.69 . STOP 4 months old: $p=1.15 \times 10^{-170}, Z=-27.85 ; 7$ months old: $p=6.48 \times 10^{-210}, Z=-30.92$. Wilcoxon rank sum test (*** $\left.p<0.001\right)$. G, Examples of G-CaMP7 fluorescence traces of RUN hyperactive and STOP hyperactive cells. Hyperactive cells were defined as those having activity rate in $\geq 10 \%$ of frames either running (for RUN hyperactive cells) or resting (for STOP hyperactive cells) period. $\boldsymbol{H}, \boldsymbol{I}$, Violin plots of proportion of RUN $(\boldsymbol{H})$ and STOP $(\boldsymbol{I})$ hyperactive cells in early (E), middle (M), late (L), and relearning (R) periods in 4- and 7-month-old WT-G-CaMP7 and AD-G-CaMP7 mice. $\boldsymbol{H}$, RUN 4 months old: $p=0.0013$ by two-way ANOVA effect of genotype, $F_{(1,186)}=10.72 ; 7$ months old: $p=3.72 \times 10^{-7}$ by two-way ANOVA effect of genotype, $F_{(1,149)}=28.31$. $\boldsymbol{I}$, STOP 4 months old: $p=7.00 \times 10^{-7}$ by two-way ANOVA effect of genotype, $F_{(1,186)}=26.39 .7$ months old: $p=2.13 \times 10^{-5}$ by two-way ANOVA effect of genotype, $F_{(1,149)}=19.28$. Two-way ANOVA followed by a Tukey-Kramer post hoc test $\left({ }^{*} p<0.05,{ }^{* *} p<0.01\right)$. J, $\boldsymbol{K}$, Cumulative histograms and violin plots of shortest distance from G7D2 aggregates to each hyperactive cells (red) in comparison with distance to all cells (black) during run ( $(J)$ and stop (K) in 4- and 7-month-old AD-G-CaMP7 mice. J, RUN 4 months old: AD = four mice (hyperactive $=16,167$ cells, nonhyperactive $=28,492$ cells); $p$ $=5.26 \times 10^{-20}, Z=-9.16 ; 7$ months old: $A D=$ four mice (hyperactive $=16,994$ cells, nonhyperactive $=23,082$ cells), $p=1.43 \times 10^{-11}, Z=-6.75 . K$, STOP 4 months old: $A D=$ four mice (hyperactive $=1939$ cells, nonhyperactive $=42,720$ cells), $p=0.0055, Z=-2.78 ; 7$ months old: $A D=$ four mice (hyperactive $=2,050$ cells, nonhyperactive $=38,026$ cells), $p=3.44 \times 10^{-20}, Z=-9.20$. Wilcoxon rank sum test $\left({ }^{* *} p<0.01,{ }^{* * *} p<0.001\right)$. 
A

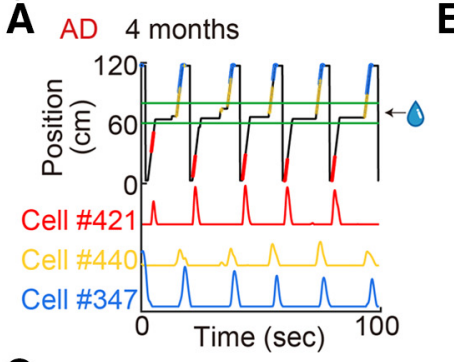

B
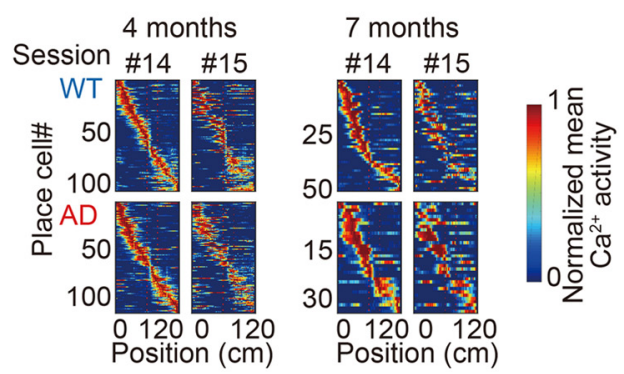

C
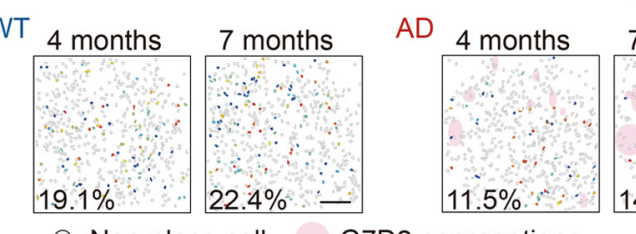

Non-place cell

E

D

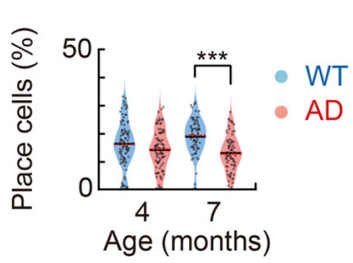

$\mathbf{F}$

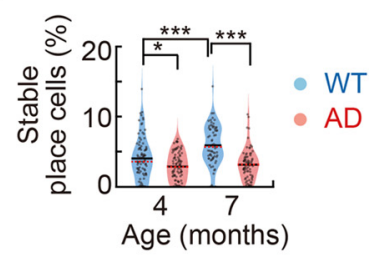

H
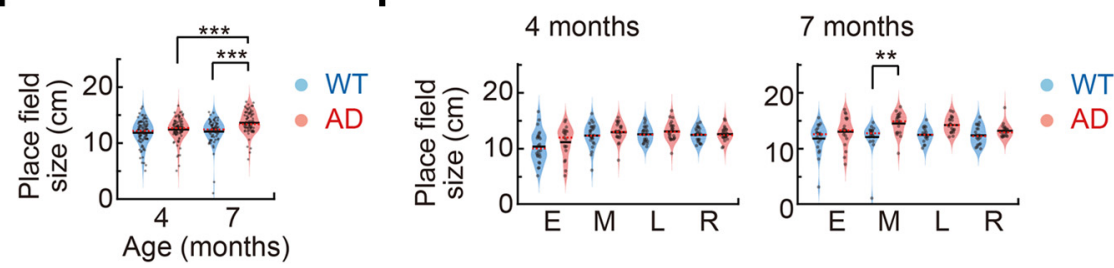

Figure 7. Age-dependent impairment of place cells in AD-G-CaMP7 mice. $A$, Examples of place cell activity. Position of a mouse and $\mathrm{Ca}^{2+}$ traces are shown. Place cell activities of cells \#421, \#440, and \#347 are color coded. $\boldsymbol{B}$, Activity heat maps of the place cells detected in session \#14 in 4- and 7-month-old WT-G-CaMP7 (top) and AD-G-CaMP7 (bottom) mice. The same cells in session \#15 are also shown. The cells are sorted by their place field positions on \#14. C, Example of the cell map of the stratum pyramidale with place cell peak positions and G7D2 aggregates with a pink ellipse from 4- and 7-month-old WT-G-CaMP7 and AD-G-CaMP7 mice. The proportions of place cells are shown (bottom left). $\boldsymbol{D}$, Violin plots of proportion of place cells among all active cells in 4- and 7-month-old WT-G-CaMP7 and AD-G-CaMP7 mice. Four months old: WT = five mice (99 sessions), AD = five mice ( 95 sessions). Seven months old: WT $=$ four mice (77 sessions), $A D=$ four mice $\left(80\right.$ sessions); $p=1.56 \times 10^{-7}$ by two-way ANOVA effect of genotype, $F_{(1,347)}=28.68 ; p=0.34$ by two-way ANOVA effect of age, $F_{(1,347)}=0.90$. $E$, Breakdown of $D$ to early $(\mathrm{E})$, middle $(\mathrm{M})$, late $(\mathrm{L})$, and relearning $(\mathrm{R})$ periods of 4- and 7-month-old WT-G-CaMP7 and AD-G-CaMP7 mice. Four months old: $p=0.068$ by two-way ANOVA effect of genotype, $F_{(1,196)}=3.37$. Seven months old: $p=1.22 \times 10^{-8}$ by twoway ANOVA effect of genotype, $F_{(1,149)}=36.38$. $\boldsymbol{F}$, Violin plots of proportion of stable place cells among all active cells in 4 - and 7-month-old WT-G-CaMP7 and AD-G-CaMP7 mice. Four months old: WT = five mice (94 sessions), AD = five mice (88 sessions). Seven months old: $W T=$ four mice (71 sessions), $A D=$ four mice (76 sessions); $p=2.06 \times 10^{-11}$ by two-way ANOVA effect of genotype, $F_{(1,325)}=48.24 ; p=1.51 \times 10^{-4}$ by two-way ANOVA effect of age, $F_{(1,325)}=14.71$. G, Breakdown of $\boldsymbol{F}$ to early $(\mathrm{E})$, middle (M), late (L), and relearning (R) periods of 4- and 7-month-old WT-G-CaMP7 and AD-G-CaMP7 mice. Four months old: $p$ $=0.0024$ by two-way ANOVA effect of genotype, $F_{(1,174)}=9.48$. Seven months old: $p=4.63 \times 10^{-11}$ by two-way ANOVA effect of genotype, $F_{(1,139)}=51.05$. $\boldsymbol{H}$, Violin plots of place field size in 4- and 7-month-old WT-G-CaMP7 and AD-G-CaMP7 mice. Four months old: $W T=$ five mice ( 99 sessions), $A D=$ five mice (94 sessions). Seven months old: WT $=$ four mice (77 sessions), $\mathrm{AD}=$ four mice $\left(80\right.$ sessions); $p=2.57 \times 10^{-6}$ by two-way ANOVA effect of genotype; $F_{(1,346)}=22.87, p=0.0016$ by twoway ANOVA effect of age, $F_{(1,346)}=10.12$. I, Breakdown of $\boldsymbol{H}$ to early $(\mathrm{E})$, middle $(\mathrm{M})$, late $(\mathrm{L})$, and relearning $(\mathrm{R})$ learning periods of 4- and 7-month-old WT-G-CaMP7 and AD-G-CaMP7 mice. Four months old: $p=0.067$ by two-way ANOVA effect of genotype, $F_{(1,185)}=3.40$. Seven months old: $p=6.34 \times 10^{-6}$ by two-way ANOVA effect of genotype, $F_{(1,149)}=21.92$. Two-way ANOVA followed by a Tukey-Kramer post hoc test $\left(^{*} p<0.05,{ }^{* *} p<0.01,{ }^{* * *} p<0.001\right)$. if it has event rate of $>10 \%$, which roughly corresponds to the top $95^{\text {th }}$ percentile value of event rate $(10.68 \%)$ seen in 4-month-old WT-G-CaMP7 mice (Fig. 6B, see above, Materials and Methods). There was an increase of the proportion of hyperactive cells starting from 4-month-old AD-G-CaMP7 mice comparable with WT-G-CaMP7 mice, and the difference became more pronounced in later ages (Fig. 6B,C). We next examined the spatial distribution of hyperactive neurons in relation to the A $\beta$ aggregates (Fig. $6 D$ ). The hyperactive neurons were located significantly closer than other cells to G7D2 aggregates at all ages (Fig. $6 E$ ). These results demonstrate that $\mathrm{A} \beta$ decreases the overall number of active neurons, while the activity of remaining neurons increase, especially near the G7D2 aggregates.

\section{Place cells are decreased in AD-G- CaMP7 mice}

It is known that hippocampal neurons show different activity depending on animals' behavior. While an animal is running, the electroencephalogram (EEG) predominantly shows theta power, and a robust neuronal activity is observed. In contrast, when an animal is stationary, the EEG shows nontheta waves with occasional sharp wave/ripple activity, and neuronal activity is overall reduced, whereas synchronicity increases. We therefore wondered whether $\mathrm{A} \beta$ has different effects on neuronal activity depending on the behavior status. We analyzed hyperactive cells separately during runand-stop phases on both fixed-distance and time tasks (Fig. 6F,G). As expected, the neuronal activity was higher in the run phase in both genotypes, but there were more hyperactive cells in AD-G-CaMP7 mice at 7 months especially during the run phase (Fig. $6 \mathrm{H}$, I). Hyperactive cells were near G7D2 aggregates regardless of behavior status (Figs. $6 E, J, K$ ).

While animals are running on the track, hippocampal place cells specifically fire according to the location of the animals. The place cells are proposed to play an important role in spatial memory formation (O'Keefe and Dostrovsky, 1971; O'Keefe and Nadel, 1978). Therefore, it is of great interest to test the properties of place cells in this animal model. We could detect place cells in the fixed-distance task of our VR system (Fig. 7A), consistent 
with previous reports (O'Keefe and Dostrovsky, 1971; Cacucci et al., 2008; Dombeck et al., 2010; Ravassard et al., 2013; Ziv et al., 2013; Zhao et al., 2014; Sato et al., 2020). Although a spatial map was formed in both WT-G-CaMP7 and AD-G-CaMP7 mice (Fig. 7B,C), the proportion of place cells significantly decreased in 7-month-old AD-G-CaMP7 mice compared with WT-G-CaMP7 (Fig. $7 D)$. It was most obvious at middle and late periods at 7 months of age (Fig. $7 E$ ).

We previously found that stability of place cells across days correlates with the cognitive value of the salient locations such as rewards and landmarks (Sato et al., 2020). We therefore tested whether the stability of the place field is altered in ADG-CaMP7 animals. For this purpose, place fields of a cell identified in a session were compared with those in the following session. In 4-month-old WT-G-CaMP7 mice, $4.1 \pm 0.3 \%$ of total neurons (Fig. $7 F$ ) or $21.7 \pm 1.2 \%$ of place cells had the same place field in the following session. In ADG-CaMP7 mice, the stability was already lower than WT-G-CaMP7 mice at 4 months (WT-G-CaMP7: $4.06 \pm 0.31 \%$; AD-G-CaMP7: $2.91 \pm 0.21 \%$ of total cells, $p=0.028)$. The ratio of stable place cells gradually increased as training proceeded in the WT-G-CaMP7 mice at 4 months of age ( 4 months, early period $1.2 \pm 0.4 \%$, late period $5.2 \pm 0.6 \%, p<0.001$ ), whereas it remained low in AD-G-CaMP7 mice ( 4 months, early period $2.0 \pm 0.7 \%$, late period $3.1 \pm 0.3 \%, p=0.86$ ) (Fig. $7 G$ ). The differences became more obvious at 7 months of age.

To compare the specificity of the information carried by the place cells, we measured the size of place field for each place cell. The place field size was larger in ADG-CaMP7 mice than in WT-G-CaMP7 mice at 7 months old (Fig. $7 H, I$ ). These results indicate that in AD-G-CaMP7 mice, place cell stability declined at first, and then the place cell number and the specificity decreased, leading to the impairment of spatial memory.

\section{Enrichment of place cells at reward location is impaired in AD-G-CaMP7 animals}

We have previously observed local enrichment of place cells at locations containing rewards or sensory cues (Sato et al., 2020). To test whether this property is impaired in AD-GCaMP7 animals, we plotted the distribution of place cells along the track. We found enrichment of place cells at the beginning and the end of the track (Fig. $8 A$ ). In addition, we found a significant enrichment of place cells in the reward zone compared with the nonrewarded zone, which is consistent with our previous study (Sato et al., 2020). This is because of the selective stabilization of the place cells near the reward zone compared with the nonrewarded zone (Fig. $8 B)$. In AD-G-CaMP7 mice, the stabilization was not observed both at 4 and 7 months old, and consequently, there was no enrichment of place cells at 7 months of age. However, although the number of stable cells were decreased in AD-G-CaMP7 mice, the position of place field was reproducible among the sessions at a level comparable to WT-GCaMP7 (Fig. 8C,D). These results indicate that the rewardinduced formation of place cell map is impaired in AD-GCaMP7 mice, leading to the reduced stability of place cell representations.

Representation of time is not affected in AD-G-CaMP7 mice In addition to the spatial information, the hippocampus bears neurons that encode elapsed time. Although time of different scales can be represented in different ways in the brain (Tsao et 
A

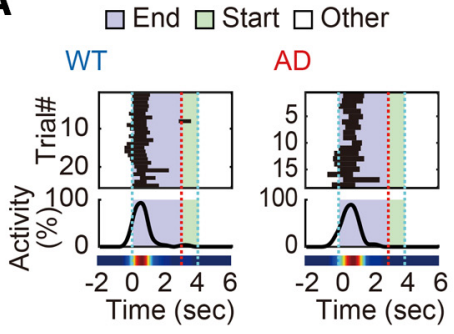

B

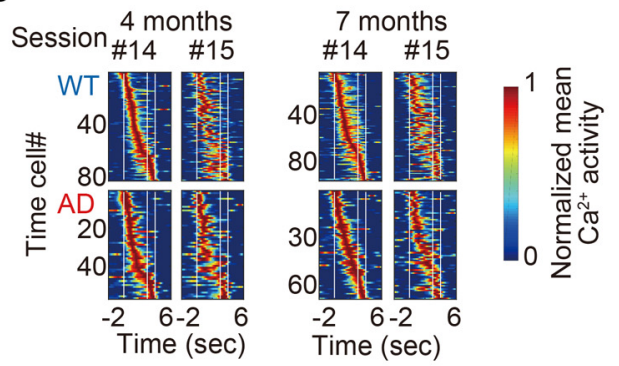

C

E

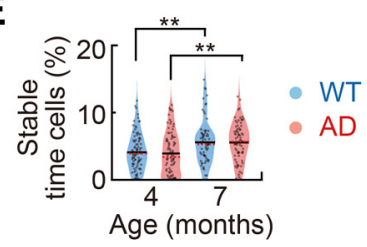

G
D

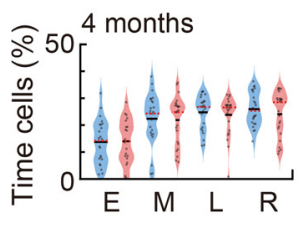

$\mathbf{F}$

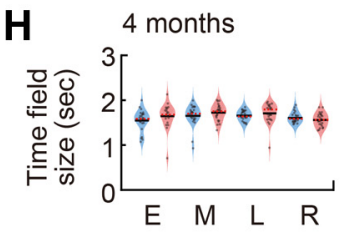

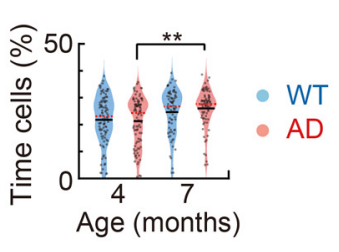
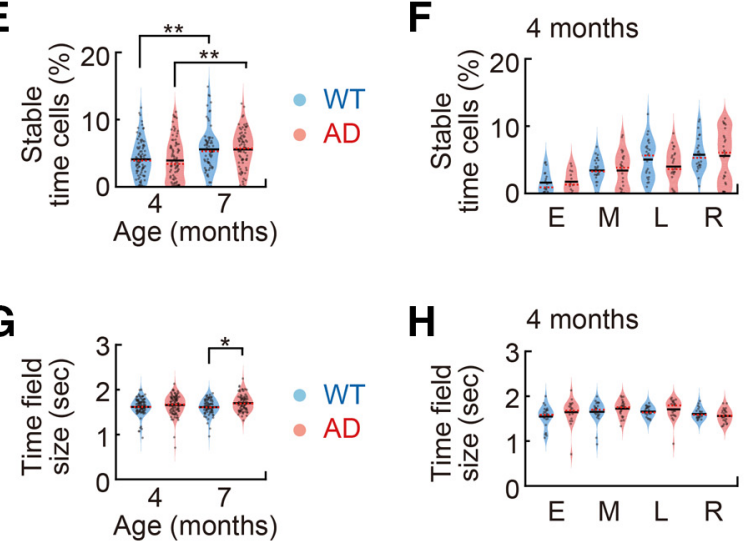

and AD-G-CaMP7 mice in all ages (Fig. $9 B)$. The fraction of time cells was comparable and gradually increased with sessions both in WT-G-CaMP7 and AD-G-CaMP7 mice (Fig. $9 C, D$ ). The stability of time cells was also comparable between genotypes with increments along sessions both in WT-G-CaMP7 and AD-G-CaMP7 mice (Fig. 9E,F). Comparison of the time window size in different ages and genotypes showed that it expanded, albeit slightly, in 7-monthold AD-G-CaMP7 mice compared with WT-G-CaMP7 mice (Fig. 9G,H), possibly indicating a sign that time cells are also impaired. Overall, however, these results indicate that the time cells are more resilient to the pathologic change in $\mathrm{A} \beta$ progression compared with place cells.

These two cell types are interchangeable, which is also crucial for shaping the cognitive map (Sato et al., 2020). Therefore, we compared the conversion of place and time cells into different cell types between adjacent sessions. Place cells tend to stay as place cells, and time cells tend to stay as time cells (Fig. 10A,B). A proportion that was smaller than the average $($ All $\rightarrow$, Fig. $10 A, B)$ was converted to other cell types, suggestive of nonrandom conversion of cell types. We observed that the stability of place cells (place cell $\rightarrow$ place cell) was lower than that of time cells (time cell $\rightarrow$ time cell) in AD-G-CaMP7 mice at 7 months. These results show that the cells maintain their cell type specificity either as a place cell or a time cell but such ability is declined with aged AD-G-CaMP7 mice, confirming the idea of altered stability of these two classes of neurons.

\section{Place cells became hyperactive and lost spatial representations near G7D2 aggregates}

Given the increase in the hyperactive cell population in the run phase in $\mathrm{AD}-\mathrm{G}$ CaMP7 mice (Fig. 6), we tested what information they carry. Among hyperactive cells, the proportion of place cells decreased in AD-G-CaMP7 mice, whereas that of time cells remained similar between genotypes (Fig. 11A), phenocopying the overall tendency of place and time cells (Figs. 7, 9). This resulted in an increase in the proportion of hyperactive nonplace/nontime cells and hyperactive time cells (Fig. 11A). We next examined the spatial distribution of al., 2018), time cells encode time on the order of seconds (MacDonald et al., 2011, 2013), possibly providing the element for perception of a longer time duration. We found a subpopulation of neurons having time-locked activities on the fixed-time task of our VR task (Fig. 9A), similar to the reported time cells in which they also fire during an empty temporal gap between the key events (MacDonald et al., 2011, 2013). These cells ( 25\% of all detected cells) were formed similarly both in WT-G-CaMP7 hyperactive and nonhyperactive place and time cells in relation to the G7D2 aggregates. At 4 months, both place and time cells were distributed similarly to each other in relation to the position of G7D2 aggregates, as well the cells which do not code either information (nonplace/nontime cells) (Fig. 11B). However, at 7 months, the place cells were distributed farther from the G7D2 aggregates than time cells or nonplace/nontime cells. In other 
words, hyperactive cells in the vicinity of the G7D2 aggregates lost spatial information. Together, these results indicate that G7D2 aggregates locally impair spatial information more preferentially than temporal encoding.

Finally, we traced the same cells from 4 to 7 months and explored the impacts of G7D2 aggregates on cellular dysfunctions. We first detected the cells in 4-month-old animals and then identified the same cells at 7 months (Fig. 11C). We calculated the stability of each cell by counting how many sessions the cell was identified as a place cell (Fig. 11D) or a time cell (Fig. $11 E)$ and segregated the cells into two groups, near $(\leq 100 \mu \mathrm{m})$ and far $(100$ $200 \mu \mathrm{m})$ from $\mathrm{A} \beta$ aggregates. The place cell at 4 months near the aggregate had a higher chance to lose spatial or temporal information and become a hyperactive nonplace/nontime cell than those far away at 7 months (Fig. 11D). This was more evident in stable place cells, which shows a higher chance of detection at 4 months. In contrast, time cells did not have such a tendency (Fig. 11E). These results revealed that place cells, especially near G7D2 aggregates, becomes hyperactive, but they cannot compensate for the function and rather lose spatial representations, whereas time cells were more stable.

\section{Discussion}

The $A p p^{N L-G-F / N L-G-F}$ mouse line (Saito et al., 2014) coexpressing G-CaMP7 and DsRed2 in pyramidal neurons under Thy1 promoter allowed us to simultaneously visualize $\mathrm{A} \beta$ aggregates (Fig. 1C) and activities of the same neuronal populations (Fig. $5 C)$ in awake mice over months without tissue staining. The aggregates colocalized with the highly aggregated $\mathrm{A} \beta$ and affected the activity of nearby cells. This provides a unique opportunity to chronically and simultaneously image the $\mathrm{A} \beta$ pathogenesis and circuit impairment in the hippocampal CA1 region.

We found that hippocampal place cells were specifically impaired in the AD-G-CaMP7 animal (Fig. 7), whereas time cells were spared (Fig. 9). The fraction, stability, and field size of place cells were significantly impaired in the AD-G-CaMP7 mice, starting at 4 months and fully manifested by 7 months old. Because there were no differences in gross motor activities such as running speed and travel distance (Fig. 4), we consider that this is because of a primary change in the properties of the cells, not secondary to a change in behavior. This is largely consistent with electrophysiological studies on APP-overexpressing mouse models (Zhao et al., 2014; Cayzac et al., 2015; Mably et al., 2017). Also, the decline of spatial cognition was reported in 6-monthold $A p p^{N L-G-F / N L-G-F}$ mice (Saito et al., 2014). Cognitively relevant locations such as reward sites slow down the turnover of the place cells (Fig. 8), which may confer stability and enhancement of the positional memory encoding of the location. The decreased stability and number of place cells seen in AD-G-CaMP7 mice may thus represent the cellular counterpart of memory impairment.

In contrast to place cells, we found the time cells were not affected by $\mathrm{A} \beta$ pathology until 7 months old. The time cells observed here reproduced the essential properties of time cells reported by others using more demanding tasks (Pastalkova et al., 2008; Kraus et al., 2013; MacDonald et al., 2013) in that they sequentially fire in the second-order temporal resolution in a manner reproducible in each lap (Fig. 9). It is generally believed that the place cells and the time cells arise from the same population of hippocampal CA1 pyramidal neurons, which differently fire depending on the task and context. This is based on the observation that the firing of a time cell itself has positional preference. Moreover, in experiments where an animal alternates between two tasks in a single session, a neuron can fire either as a place cell or a time cell (Kraus et al., 2013; Eichenbaum, 2014; Haimerl et al., 2019). Given that these neurons arise from the same population of cells, coupled with the specific deficits we observed in positional but not temporal coding, this suggests a circuit mechanism underlying the formation of these two cell types, such as input specificity, which may explain the differential vulnerability of these cells to $\mathrm{A} \beta$ pathology (Fig. 10). Although place cells are formed as an integration of external and internal sensory input, time cells do not require external input except for cues indicating the start and end of events. The spatial information is transmitted from the medial entorhinal cortex (MEC) to the hippocampus (Brun et al., 2002; Brandon et al., 2014; Jun et al., 2020). Indeed, in these knock-in mice, MEC grid cells formation is significantly impaired in the presence of external cues (Jun et al., 2020). In contrast, the time cells are independent of MEC inputs (Sabariego et al., 2019); rather, an input from the prefrontal cortex area is implicated (Kim et al., 2013). Also, a selective inactivation of the medial septum affects place cells but not time cells (Wang et al., 2015). However, we do not rule out the possibility that the time cells also show impairment if a task becomes more complex, for example, by making the task context dependent (Sabariego et al., 2019). 
A

Hyperactive

non-place/non-time cells
Hyperactive place cells
Hyperactive time cells

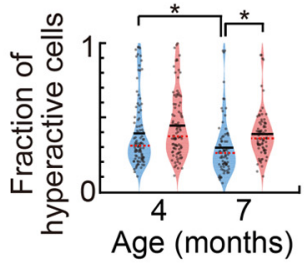

B

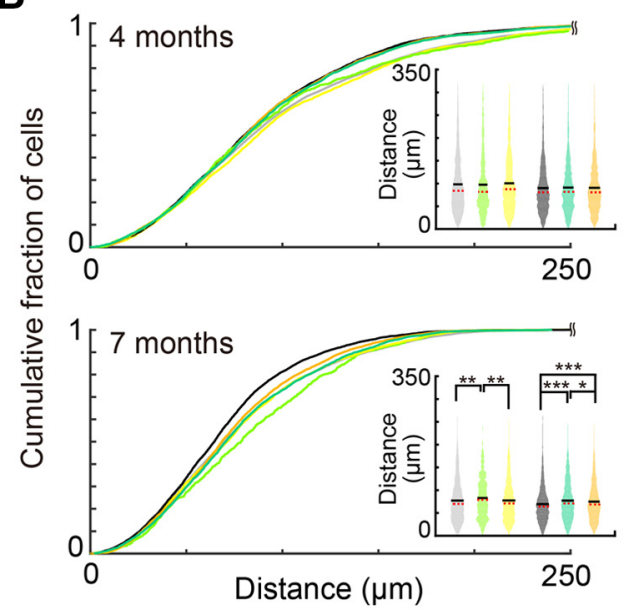

C
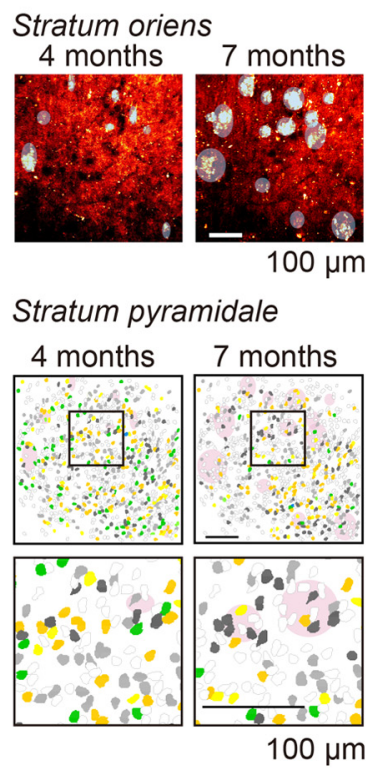

- WT

- $A D$
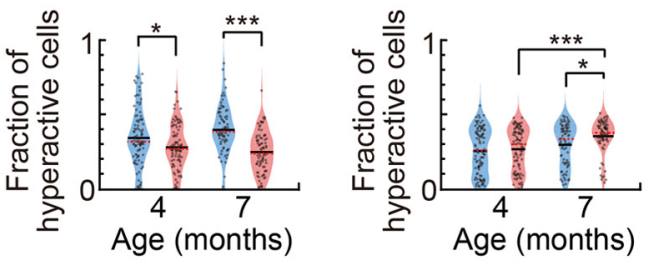

G7D2

aggregates

o Inactive cell

Hyperactive

- Non-place/

non-time cell

- Place cell

- Time cell

- Place/time cell

Non-hyperactive

- Non-place/

non-time cell

- Place cell

- Time cell

- Place/time cell

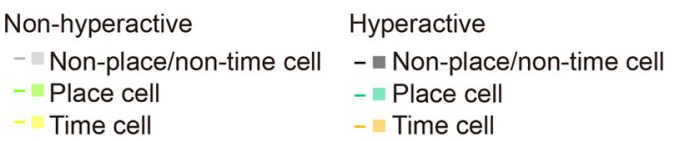

D

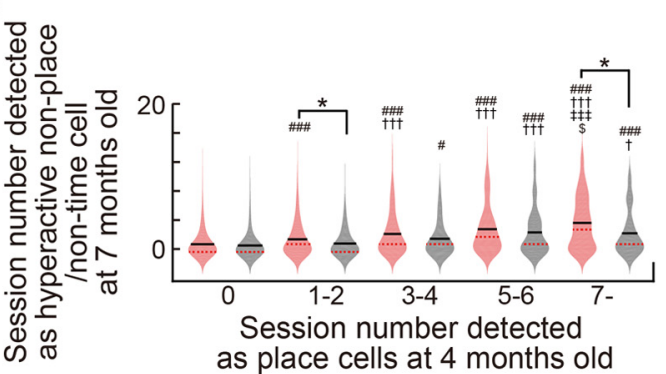

E

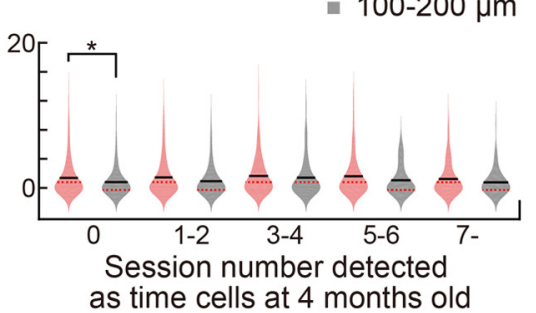

Figure 11. Place cells lost spatial information near G7D2 aggregates but not time cells. $A$, Violin plots of proportion of hyperactive nonplace/nontime cells, hyperactive place cells, hyperactive time cells, and hyperactive place/time cells among hyperactive cells in 4- and 7-month-old AD-G-CaMP7 and WT-G-CaMP7 mice. Four months old: WT = five mice (99 sessions), AD = five mice (95 sessions). Seven months old: WT $=$ four mice (77 sessions), $A D=$ four mice (80 sessions). Hyperactive nonplace/nontime cell: $p=0.0012$ by two-way ANOVA effect of genotype, $F_{(1,347)}=10.73 ; p=5.60 \times 10^{-4}$ by two-way ANOVA effect of age, $F_{(1,347)}=12.13$. Hyperactive place cell: $p=2.80 \times 10^{-9}$ by two-way ANOVA effect of genotype, $F_{(1,347)}=37.23 ; p=$ 0.52 by two-way ANOVA effect of age, $F_{(1,347)}=0.42$. Hyperactive time cell: $p=0.018$ by two-way ANOVA effect of genotype, $F_{(1,347)}=5.64 ; p=1.57 \times 10^{-5}$ by two-way ANOVA effect of age, $F_{(1,347)}=19.19$. $\boldsymbol{B}$, Cumulative histograms and violin plots of shortest distance from G7D2 aggregates to nonhyperactive nonplace/nontime cells (light gray), nonhyperactive place cells (light green), nonhyperactive time cells (yellow), hyperactive nonplace/nontime cells (dark gray), hyperactive place cells (green), and hyperactive time cells (orange) in 4- and 7-month-old WTG-CaMP7 and AD-G-CaMP7 mice. Hyperactive place cells are far from G7D2 aggregates. AD = four; four mice in 4- and 7-month-old nonhyperactive nonplace/nontime cell = 22,407, 18,446 cells; nonhyperactive place cell = 1,288, 903 cells; nonhyperactive time cell 4,928, 3,783 cells Hyperactive nonplace/nontime cell = 5,998, 6,529 cells; hyperactive place cell = 5,257, 4,427 cells; hyperactive time cell $=5,553,6,560$ cells. Four months old: $p=0.070$ by two-way ANOVA effect of cell type, $F_{(2,45425)}=2.67$. Seven months old: $p=1.35 \times 10^{-17}$ by two-way ANOVA effect of cell type, $F_{(2,40642)}=38.88$. C, Example of the maximum intensity projection image at the stratum oriens and the cell map of the stratum pyramidale with cell type indicated with green (hyperactive place cell), light green (nonhyperactive place cell), orange (hyperactive time cell), yellow (nonhyperactive time cell), blue (hyperactive place/time cell), cyan (nonhyperactive place/ time cell), dark gray (hyperactive nonplace/nontime cell), light gray (nonhyperactive nonplace/nontime cell), and white (inactive cell) dots and G7D2 aggregates with pink ellipses from 4- and 7-month-old AD-G-CaMP7 mice. $\boldsymbol{D}, \boldsymbol{E}$, Violin plots showing the number of sessions of 20 at 4 months where the cells were detected as place cells $(\boldsymbol{D})$ or time cells $(\boldsymbol{E})$ and the number of sessions at 7 months where the cells were detected as hyperactive nonplace/nontime cells. The cells were segregated $\leq 00 \mu \mathrm{m}$ (red) or 100-200 $\mu \mathrm{m}$ (gray) from the A $\beta$ aggregate (at 7 months old). The cells with higher chance of becoming place cells had a higher chance of becoming a hyperactive nonplace/nontime cells at 7 months. The cells near the aggregate had larger chance of becoming hyperactive nonplace/nontime cells. Such a tendency was not present in time cells. $\boldsymbol{D}, \mathrm{AD}=$ four mice; $100-200 \mu \mathrm{m}(n=398,219,79,51,37 \mathrm{cells}), \leq 100 \mu \mathrm{m}(n=1204,644$, $316,170,136$ cells); $p=2.70 \times 10^{-7}$ by two-way ANOVA effect of distance, $F_{(1,3244)}=26.57 ; p=3.40 \times 10^{-46}$ by two-way ANOVA effect of session number, $F_{(4,3244)}=56.57$. $E$, AD $=$ four mice; 100-200 $\mu \mathrm{m}\left(n=234,241,135,85,89\right.$ cells), $\leq 100 \mu \mathrm{m}\left(n=731,775,475,224,265\right.$ cells); $p=1.33 \times 10^{-5}$ by two-way ANOVA effect of distance, $F_{(1,3244)}=19.02 ; p=0.019$ by two-way ANOVA effect of session number, $F_{(4,3244)}=2.96$. Two-way ANOVA followed by a Tukey-Kramer post hoc test (\#: versus $0 ;$;: versus $1-2 ; \neq$ : versus $3-4 ; \$$ : versus $5-6$; ${ }^{*} p<0.05$, $\left.{ }^{* *} p<0.01,{ }^{* * *} p<0.001\right)$. 
We found a reduction in the number of active neurons (Fig. $5 D)$. Before that occurred, we found that a subset of cells became hyperactive near $\mathrm{A} \beta$ (Fig. 6). This is consistent with a previous study that found that hyperactive cells were linked with $\mathrm{A} \beta$ depositions in the hippocampus (Busche et al., 2008). We additionally revealed the elevated hyperactive cells in awake AD mice. The relationship between neuronal hyperactivity and $\mathrm{A} \beta$ depositions was also shown in AD patients (Sperling et al., 2009).

The relationship among the inactivity of neurons, the hyperactivity of the remaining cells, and $\mathrm{A} \beta$ aggregates is not known at this point. It has been generally conceived that $\mathrm{A} \beta$ aggregates are less toxic than freely floating amyloid. As opposed to this, we observed cells near the aggregates that are hyperactive and lose spatial information. This could be a direct effect of $\mathrm{A} \beta$ aggregates on the function of neurons in the vicinity. Alternatively, it could be because of $\mathrm{A} \beta$ oligomers, which are associated with the amyloid plaques as visualized by an oligomer-specific antibody (Lin et al., 2007; Shankar et al., 2009). In either case, our results propose that cells are more severely affected in the vicinity of A $\beta$ plaque.

$\mathrm{A} \beta$ is known to reduce synaptic transmission and plasticity (Shankar et al., 2007; Keskin et al., 2017; He et al., 2019). On the other hand, the hyperactivity of remaining neurons can be a direct effect of $\mathrm{A} \beta$, for example, via inhibition of glutamate reuptake (Zott et al., 2019) or an indirect effect such as the dysfunction of inhibitory neurons by $\mathrm{A} \beta$ or homeostatic scaling. The A $\beta$ peptide is released from neurons activity-dependently (Yamamoto et al., 2015). This support the idea of an aggravating cycle; $\mathrm{A} \beta$ peptide release $\rightarrow$ neuronal hyperactivity increases near the $\mathrm{A} \beta$ aggregate $\rightarrow$ more $\mathrm{A} \beta$ peptide release (Zott et al., 2019). Moreover, we suggest that hyperactivity could be one reason that spatial dysfunction occurs. Previously, it was demonstrated that hyperactive neurons lose orientation and direction selectivity in the sensory cortex of APP $23 \times$ PS45 mice (Grienberger et al., 2012). In this study, we show that cells near $\mathrm{A} \beta$ aggregates became hyperactive and concomitantly lost spatial information, indicating the relationships between hyperactivity and spatial memory dysfunctions. Indeed, $\mathrm{A} \beta$-dependent impairment of slow-wave propagation, related to the breakdown of slow-wave activity, was rescued by enhancing GABAergic inhibition in the AD mouse model (Busche et al., 2015). The effect on place cells by suppressing neuronal activity should also be explored. The technique in this study can be thus beneficial for drug assessment in the evaluation of cellular dysfunctions and $\mathrm{A} \beta$ depositions in each stage of $\mathrm{AD}$.

\section{References}

Berkowitz LE, Harvey RE, Drake E, Thompson SM, Clark JB (2018) Progressive impairment of directional and spatially precise trajectories by TgF344-Alzheimer's disease rats in the morris water task. Scientific Reports 8:16153.

Brandon MP, Koenig J, Leutgeb S (2014) Parallel and convergent processing in grid cell, head-direction cell, boundary cell, and place cell networks. Wiley Interdiscip Rev Cogn Sci 5:207-219.

Brun VH, Otnass MK, Molden S, Steffenach H-A, Witter MP, Moser M-B, Moser EI (2002) Place cells and place recognition maintained by direct entorhinal-hippocampal circuitry. Science 296:2243-2246.

Busche MA, Eichhoff G, Adelsberger H, Abramowski D, Wiederhold K, Haass C, Staufenbiel M, Konnerth A, Garaschuk O (2008) Clusters of hyperactive neurons near amyloid plaques in a mouse model of Alzheimer's disease. Science 321:1686-1690.

Busche MA, Kekuš M, Adelsberger H, Noda T, Förstl H, Nelken I, Konnerth A (2015) Rescue of long-range circuit dysfunction in Alzheimer's disease models. Nat Neurosci 18:1623-1630.
Cacucci F, Yi M, Wills TJ, Chapman P, O'Keefe J (2008) Place cell firing correlates with memory deficits and amyloid plaque burden in Tg2576 Alzheimer mouse model. Proc Natl Acad Sci U S A 105:7863-7868.

Carrasco MC, Guillem MJ, Redolat R (2000) Estimation of short temporal intervals in Alzheimer's disease. Exp Aging Res 26:139-151.

Cayzac S, Mons N, Ginguay A, Allinquant B, Jeantet Y, Cho YH (2015) Altered hippocampal information coding and network synchrony in APP-PS1 mice. Neurobiol Aging 36:3200-3213.

Dombeck DA, Harvey CD, Tian L, Looger LL, Tank DW (2010) Functional imaging of hippocampal place cells at cellular resolution during virtual navigation. Nat Neurosci 13:1433-1440.

Eichenbaum H (2014) Time cells in the hippocampus: a new dimension for mapping memories. Nat Rev Neurosci 15:732-744.

Ergorul C, Eichenbaum H (2004) The hippocampus and memory for "what, where," and "when." Learn Mem 11:397-405.

Grienberger C, Rochefort NL, Adelsberger H, Henning HA, Hill DN, Reichwald J, Staufenbiel M, Konnerth A (2012) Staged decline of neuronal function in vivo in an animal model of Alzheimer's disease. Nat Commun 3:710-774.

Haimerl C, Angulo-Garcia D, Villette V, Reichinnek S, Torcini A, Cossart R, Malvache A (2019) Internal representation of hippocampal neuronal population spans a time-distance continuum. Proc Natl Acad Sci U S A 116:7477-7482.

He Y, Wei M, Wu Y, Qin H, Li W, Ma X, Cheng J, Ren J, Shen Y, Chen Z, Sun B, Huang F, Shen Y, Zhou Y (2019) Amyloid $\beta$ oligomers suppress excitatory transmitter release via presynaptic depletion of phosphatidylinositol-4,5-bisphosphate. Nat Commun 10:1193

Jun H, Bramian A, Soma S, Saito T, Saido TC, Igarashi KM (2020) Disrupted place cell remapping and impaired grid cells in a knockin model of Alzheimer's disease. Neuron 107:1095-1112.

Kaneko H, Kakita A, Kasuga K, Nozaki H, Ishikawa A, Miyashita A, Kuwano R, Ito G, Iwatsubo T, Takahashi H, Nishizawa M, Onodera O, Sisodia SS, Ikeuchi T (2007) Enhanced accumulation of phosphorylated $\alpha$-synuclein and elevated $\beta$-amyloid $42 / 40$ ratio caused by expression of the presenilin-1 $\Delta \mathrm{T} 440$ mutant associated with familial lewy body disease and variant Alzheimer's disease. J Neurosci 27:13092-13097.

Keskin AD, Kekuš M, Adelsberger H, Neumann U, Shimshek DR, Song B, Zott B, Peng T, Förstl H, Staufenbiel M, Nelken I, Sakmann B, Konnerth A, Busche MA (2017) BACE inhibition-dependent repair of Alzheimer's pathophysiology. Proc Natl Acad Sci U S A 114:8631-8636.

Kim J, Ghim J, Lee JH, Jung MW (2013) Neural correlates of interval timing in rodent prefrontal cortex. J Neurosci 33:13834-13847.

Kraus B, Robinson R, White J, Eichenbaum H, Hasselmo M (2013) Hippocampal "time cells": time versus path integration. Neuron 78:10901101.

Lin C-Y, Gurlo T, Kayed R, Butler AE, Haataja L, Glabe CG, Butler PC (2007) Toxic human islet amyloid polypeptide (h-IAPP) oligomers are intracellular, and vaccination to induce anti-toxic oligomer antibodies does not prevent h-IAPP-induced $\beta$-cell apoptosis in h-IAPP transgenic mice. Diabetes 56:1324-1332.

Mably AJ, Gereke BJ, Jones DT, Colgin LL (2017) Impairments in spatial representations and rhythmic coordination of place cells in the $3 \mathrm{xTg}$ mouse model of Alzheimer's disease. Hippocampus 27:378-392.

MacDonald CJ, Lepage KQ, Eden UT, Eichenbaum H (2011) Hippocampal "time cells" bridge the gap in memory for discontiguous events. Neuron 71:737-749.

MacDonald CJ, Carrow S, Place R, Eichenbaum H (2013) Distinct hippocampal time cell sequences represent odor memories in immobilized rats. J Neurosci 33:14607-14616.

Manita S, Suzuki T, Homma C, Matsumoto T, Odagawa M, Yamada K, Ota $\mathrm{K}$, Matsubara $\mathrm{C}$, Inutsuka $\mathrm{A}$, Sato $\mathrm{M}$, Ohkura $\mathrm{M}$, Yamanaka $\mathrm{A}$, Yanagawa Y, Nakai J, Hayashi Y, Larkum ME, Murayama M (2015) A top-down cortical circuit for accurate sensory perception. Neuron 86:1304-1316.

McQuail JA, Dunn AR, Stern Y, Barnes CA, Kempermann G, Rapp PR, Kaczorowski CC, Foster TC (2021) Cognitive reserve in model systems for mechanistic discovery: the importance of longitudinal studies. Front Aging Neurosci 12:607685.

Miri A, Daie K, Burdine RD, Aksay E, Tank DW (2011) Regression-based identification of behavior-encoding neurons during large-scale optical imaging of neural activity at cellular resolution. J Neurophysiol 105:964980. 
Moodley K, Minati L, Contarino V, Prioni S, Wood R, Cooper R, D'Incerti L, Tagliavini F, Chan D (2015) Diagnostic differentiation of mild cognitive impairment due to Alzheimer's disease using a hippocampus-dependent test of spatial memory. Hippocampus 25:939-951.

Morris RG, Garrud P, Rawlins JN, O'Keefe J (1982) Place navigation impaired in rats with hippocampal lesions. Nature 297:681-683.

Muntané G, Dalfó E, Martinez A, Ferrer I (2008) Phosphorylation of tau and $\alpha$-synuclein in synaptic-enriched fractions of the frontal cortex in Alzheimer's disease, and in Parkinson's disease and related $\alpha$-synucleinopathies. Neuroscience 152:913-923.

O'Keefe J, Dostrovsky J (1971) The hippocampus as a spatial map. Preliminary evidence from unit activity in the freely-moving rat. Brain Res 34:171-175.

O'Keefe J, Nadel L (1978) The hippocampus as a cognitive map. Oxford University Press: Oxford, UK.

Ohkura M, Sasaki T, Sadakari J, Gengyo-Ando K, Kagawa-Nagamura Y, Kobayashi C, Ikegaya Y, Nakai J (2012) Genetically encoded green fluorescent $\mathrm{Ca}^{2+}$ indicators with improved detectability for neuronal $\mathrm{Ca}^{2+}$ signals. PLoS One 7:e51286.

Pastalkova E, Itskov V, Amarasingham A, Buzsáki G (2008) Internally generated cell assembly sequences in the rat hippocampus. Science 321:13221328.

Ravassard P, Kees A, Willers B, Ho D, Aharoni D, Cushman J, Aghajan ZM, Mehta MR (2013) Multisensory control of hippocampal spatiotemporal selectivity. Science 340:1342-1346.

Sabariego M, Schönwald A, Boublil BL, Clark RE, Leutgeb JK, Leutgeb S, Boublil BL, Zimmerman DT, Ahmadi S, Gonzalez N, Leibold C, Clark RE, Leutgeb JK, Leutgeb S (2019) Time cells in the hippocampus are neither dependent on medial entorhinal cortex inputs nor necessary for spatial working memory. Neuron 102:1235-1248.

Saito T, Matsuba Y, Mihira N, Takano J, Nilsson P, Itohara S, Iwata N, Saido TC (2014) Single App knock-in mouse models of Alzheimer's disease. Nat Neurosci 17:661-663.

Sakaguchi T, Ishikawa D, Nomura H, Matsuki N, Ikegaya Y (2012) Normal learning ability of mice with a surgically exposed hippocampus. Neuroreport 23:457-461.

Sasaguri H, Nilsson P, Hashimoto S, Nagata K, Saito T, Strooper BD, Hardy J, Vassar R, Winblad B, Saido TC (2017) APP mouse models for Alzheimer's disease preclinical studies. EMBO J 36:2473-2487.

Sato K, Higuchi M, Iwata N, Saido TC, Sasamoto K (2004) Fluoro-substituted and 13C-labeled styrylbenzene derivatives for detecting brain amyloid plaques. Eur J Med Chem 39:573-578.

Sato M, Kawano M, Ohkura M, Gengyo-ando K, Nakai J, Hayashi Y (2015) Generation and imaging of transgenic mice that express G-CaMP7 under a tetracycline response element. PLoS One 10:e0125354.
Sato M, Kawano M, Mizuta K, Islam T, Lee MG, Hayashi Y (2017) Hippocampus-dependent goal localization by head-fixed mice in virtual reality. eNeuro 4:e0369-16.

Sato M, Mizuta K, Islam T, Kawano M, Sekine Y, Takekawa T, GomezDominguez D, Schmidt A, Wolf F, Kim K, Yamakawa H, Ohkura M, Lee MG, Fukai T, Nakai J, Hayashi Y (2020) Distinct mechanisms of overrepresentation of landmarks and rewards in the hippocampus. Cell Rep 32:107864.

Shankar GM, Bloodgood BL, Townsend M, Walsh DM, Selkoe DJ, Sabatini BL (2007) Natural oligomers of the Alzheimer amyloid- $\beta$ protein induce reversible synapse loss by modulating an NMDA-type glutamate receptor-dependent signaling pathway. J Neurosci 27:2866-2875.

Shankar GM, Leissring MA, Adame A, Sun X, Spooner E, Masliah E, Selkoe DJ, Lemere CA, Walsh DM (2009) Neurobiology of disease biochemical and immunohistochemical analysis of an Alzheimer's disease mouse model reveals the presence of multiple cerebral $\mathrm{A} \beta$ assembly forms throughout life. Neurobiol Dis 36:293-302.

Sperling RA, Laviolette PS, O'Keefe K, O’Brien J, Rentz DM, Pihlajamaki M, Marshall G, Hyman BT, Selkoe DJ, Hedden T, Buckner RL, Becker JA, Johnson KA (2009) Amyloid deposition is associated with impaired default network function in older persons without dementia. Neuron 63:178-188.

Takekawa T, Asai H, Ohkawa N, Nomoto M, Okubo-Suzuki R, Ghandour K, Sato M, Hayashi Y, Inokuchi K, Fukai T (2017) Automatic sorting system for large scale calcium imaging data. bioRxiv. doi: 10.1101/215145.

Tsao A, Sugar J, Lu L, Wang C, Knierim JJ, Moser M, Moser EI (2018) Integrating time from experience in the lateral entorhinal cortex. Nature 561:57-62.

Wang Y, Romani S, Lustig B, Leonardo A, Pastalkova E (2015) Theta sequences are essential for internally generated hippocampal firing fields. Nat Neurosci 18:282-288.

Yamamoto K, Tanei Z, Hashimoto T, Wakabayashi T, Okuno H, Naka Y, Yizhar O, Fenno LE, Fukayama M, Bito H, Cirrito JR, Holtzman DM, Deisseroth K, Iwatsubo T (2015) Chronic optogenetic activation augments $\mathrm{A} \beta$ pathology in a mouse model of Alzheimer disease. Cell Rep 11:859-865.

Zhao R, Fowler SW, Chiang ACA, Ji D, Jankowsky JL (2014) Impairments in experience-dependent scaling and stability of hippocampal place fields limit spatial learning in a mouse model of Alzheimer's disease. Hippocampus 24:963-978.

Ziv Y, Burns LD, Cocker ED, Hamel EO, Ghosh KK, Kitch LJ, Gamal AE, Schnitzer MJ (2013) Long-term dynamics of CA1 hippocampal place codes. Nat Neurosci 16:264-266.

Zott B, Simon MM, Hong W, Unger F, Chen-Engerer H-J, Frosch MP, Sakmann B, Walsh DM, Konnerth A (2019) A vicious cycle of $\beta$ amyloid-dependent neuronal hyperactivation. Science 365:559-565. 